\title{
High-Frequency EPR and ENDOR Spectroscopy on Semiconductor Quantum Dots
}

\author{
Pavel G. Baranov • Sergei B. Orlinskii • \\ Celso de Mello Donegá · Jan Schmidt
}

Received: 9 March 2010/Published online: 18 July 2010

(C) The Author(s) 2010. This article is published with open access at Springerlink.com

\begin{abstract}
It is shown that high-frequency electron paramagnetic resonance (EPR) and electron-nuclear double resonance (ENDOR) spectroscopy are excellent tools for the investigation of the electronic properties of semiconductor quantum dots (QDs). The great attractions of these techniques are that, in contrast to optical methods, they allow the identification of the dopants and provide information about the spatial distribution of the electronic wave function. This latter aspect is particularly attractive because it allows for a quantitative measurement of the effect of confinement on the shape and properties of the wave function. In this contribution EPR and ENDOR results are presented on doped ZnO QDs. Shallow donors (SDs), related to interstitial $\mathrm{Li}$ and $\mathrm{Na}$ and substitutional $\mathrm{Al}$ atoms, have been identified in this material by pulsed high-frequency EPR and ENDOR spectroscopy. The shallow character of the wave function of the donors is evidenced by the multitude of ENDOR transitions of the ${ }^{67} \mathrm{Zn}$ nuclear spins and by the hyperfine interaction of the ${ }^{7} \mathrm{Li},{ }^{23} \mathrm{Na}$ and ${ }^{27} \mathrm{Al}$ nuclear spins that are much smaller than for atomic lithium, sodium and aluminium. The EPR signal of an exchange-coupled pair consisting of a shallow donor and a deep Na-related acceptor has been identified in $\mathrm{ZnO}$ nanocrystals with radii smaller than $1.5 \mathrm{~nm}$. From ENDOR experiments it is concluded that the deep Na-related acceptor is located at the interface of the $\mathrm{ZnO}$ core and the $\mathrm{Zn}(\mathrm{OH})_{2}$ capping layer, while the shallow donor is in the $\mathrm{ZnO}$ core. The spatial

P. G. Baranov

A.F. Ioffe Physical-Technical Institute, Russian Academy of Sciences, St. Petersburg 194021, Russia

S. B. Orlinskii $(\bowtie)$

Federal Center of Shared Facilities of Kazan State University, Kazan 420008, Russia

e-mail: Sergei.Orlinskii@ksu.ru

C. de Mello Donegá

Debye Institute for Nanomaterials Science, Utrecht University, 3508 TA Utrecht, The Netherlands

J. Schmidt

Huygens Laboratory, Leiden University, 2300 RA Leiden, The Netherlands
\end{abstract}


distribution of the electronic wave function of a shallow donor in $\mathrm{ZnO}$ semiconductor QDs has been determined in the regime of quantum confinement by using the nuclear spins as probes. Hyperfine interactions as monitored by ENDOR spectroscopy quantitatively reveal the transition from semiconductor to molecular properties upon reduction of the size of the nanoparticles. In addition, the effect of confinement on the $g$-factor of SDs in $\mathrm{ZnO}$ as well as in CdS QDs is observed. Finally, it is shown that an almost complete dynamic nuclear polarization (DNP) of the ${ }^{67} \mathrm{Zn}$ nuclear spins in the core of $\mathrm{ZnO}$ QDs and of the ${ }^{1} \mathrm{H}$ nuclear spins in the $\mathrm{Zn}(\mathrm{OH})_{2}$ capping layer can be obtained. This DNP is achieved by saturating the EPR transition of SDs present in the QDs with resonant high-frequency microwaves at low temperatures. This nuclear polarization manifests itself as a hole and an antihole in the EPR absorption line of the SD in the QDs and a shift of the hole (antihole). The enhancement of the nuclear polarization opens the possibility to study semiconductor nanostructures with nuclear magnetic resonance techniques.

\section{Introduction}

The intentional introduction of impurities is fundamental for the control of the electronic and optical properties of bulk semiconductors, and has led to a myriad of technological applications. These successes have stimulated similar efforts to dope colloidal semiconductor nanocrystals. The remarkable and attractive feature of colloidal semiconductor nanocrystals is that, owing to their nanoscale dimensions, size effects can be fully exploited to tailor the material properties [1]. Quantum confinement effects become increasingly important as the dimensions of the nanocrystal decrease below a certain critical limit (viz., the spatial extension of the electron wave function in the material), leading to size- and shape-dependent electronic structure. Further, as the size of a nanocrystal decreases, the surface-tovolume ratio increases dramatically. This has important consequences, one of them being that the nanocrystal becomes easily dispersible in solvents (i.e., stable colloidal suspensions can be obtained), making fabrication and processing in solution possible, which is an essential advantage of colloidal nanocrystals over nanomaterials prepared by other techniques. Besides, colloidal chemistry methods are cheaper and easier to upscale, and are also highly versatile in terms of composition, size, shape and surface control. The potential of doped colloidal semiconductor nanocrystals has spurred an intense research activity over the past decades. Unfortunately, the efforts to dope semiconductor nanocrystals in a controlled way were a limited success [2]. One of the difficulties in the field is that the optical spectroscopic techniques, that have been mainly used to study doped nanocrystals, fail to identify the chemical nature of the dopants and their location in the nanocrystal.

In this review we will present the results of electron paramagnetic resonance (EPR) and electron-nuclear double resonance (ENDOR) studies on colloidal $\mathrm{ZnO}$ nanocrystals doped with shallow donors (SD) and deep acceptors. We will show that these two magnetic resonance techniques are methods of choice to identify dopants 
in these nanocrystals and to obtain information about their electronic properties that remain hidden for optical spectroscopic techniques.

$\mathrm{ZnO}$ with a direct band gap of 3.3-3.4 eV attracts considerable attention [3-12]. The attraction of $\mathrm{ZnO}$ quantum dots is that the confinement of the electronic wave function allows the tuning of the optical and electronic properties. The effect of confinement on the electronic energy levels can easily be made visible by the change in the optical absorption spectra. The high free-exciton binding energy of $60 \mathrm{meV}$ makes excitons stable at room temperature. This binding energy increases further in confined systems resulting in a luminescence efficiency of $\mathrm{ZnO}$ nanocrystals that is much higher than that of bulk $\mathrm{ZnO}$ crystals. Doped $\mathrm{ZnO}$ nanocrystals, which can be easily processed at temperatures much lower than those for bulk $\mathrm{ZnO}$ crystals, are of particular interest because of their potential for use in light-emitting devices.

The fabrication of nanocomposites by combining $\mathrm{ZnO}$ nanocrystals and conjugated polymers is an attractive field in organic optoelectronics because the efficient luminescence from both materials is combined. The expectation is that these materials can be used in forward flat panel displays and in lighting applications and may allow the realization of photo-induced charge transfer in organic-inorganic hybrids for photovoltaic applications [13].

The group III elements (Al, Ga and In) are expected to form shallow donors in single crystals of $\mathrm{ZnO}$ by replacing $\mathrm{Zn}$ atoms. Indeed, by using EPR spectroscopy, In and $\mathrm{Ga}$ shallow donors were identified on the basis of the resolved hyperfine (HF) structure in the EPR lines [10, 14-16]. However, since such a HF splitting is absent in the EPR signals of Al-doped $\mathrm{ZnO}$ single crystals, the identity of $\mathrm{Al}$ as the core of the shallow donor has not been unambiguously ascertained.

As yet, Al, Ga and In have not been identified as shallow donors in $\mathrm{ZnO}$ nanocrystals. Stimulated by the results of high-frequency EPR and ENDOR investigations that allowed the identification of shallow donors in bulk wide-bandgap semiconductors, such as $\mathrm{AgCl}, \mathrm{ZnO}$ and $\mathrm{AlN}$, and the spatial distribution of their wave functions [17-19], we have decided to apply these techniques to doped semiconductor nanocrystals. In this contribution, we report the results obtained on $\mathrm{ZnO}$ nanocrystals. The reason to choose this material is motivated to a large extent by the ease with which $\mathrm{ZnO}$ nanocrystals can be produced by colloidal chemistry methods.

The first, rather unexpected, result of our EPR and ENDOR investigations is that interstitial $\mathrm{Li}$ and $\mathrm{Na}$ form shallow donors in $\mathrm{ZnO}$ nanocrystals [3]. The identification was based on the observation of ENDOR signals of the ${ }^{7} \mathrm{Li}(I=3 / 2)$ and ${ }^{23} \mathrm{Na}(I=3 / 2)$ spins in Li-doped and Na-doped $\mathrm{ZnO}$ nanocrystals. These observations demonstrate the attraction of EPR and ENDOR spectroscopy for studying semiconductor nanostructures, since here traditional methods of measuring n-and p-type conductivity as applied to bulk semiconductors cannot be used.

The identification of $\mathrm{Li}$ and $\mathrm{Na}$ as interstitial shallow donors in $\mathrm{ZnO}$ nanocrystals is of fundamental interest, but is probably not of great importance for practical applications in devices where one needs to control the concentration of donor impurities. For this purpose one would prefer to dope the particles with substitutional impurities like $\mathrm{Al}$, Ga or In. By applying our EPR and ENDOR 
techniques to $\mathrm{ZnO}$ nanoparticles loaded with $\mathrm{Al}$ we were able to show that $\mathrm{Al}$ forms a shallow substitutional donor in $\mathrm{ZnO}$ nanoparticles. This identification was ascertained by the EPR signal of this donor, the ENDOR signals of the ${ }^{27} \mathrm{Al}$ nuclear spin and the ENDOR signal of a multitude of ${ }^{67} \mathrm{Zn}$ nuclear spins.

A prerequisite for the observation of the EPR signal of the unpaired spin of shallow donors at liquid-helium temperatures is that the $\mathrm{ZnO}$ quantum dots (QDs) are first illuminated with above-band-gap light. This observation shows that there must be deep acceptors present in the nanocrystals that capture the thermally excited donor electrons at room temperature. Apparently these electrons remain frozen at the acceptor when the material is cooled in the dark to low temperature. The aboveband-gap light transfers the electron from the acceptor to the donor and makes both sites paramagnetic.

The combination of EPR and optical experiments allowed us to demonstrate [7] that donor-acceptor pairs are formed in the confined structure of $\mathrm{ZnO}$ nanoparticles between the shallow, interstitial Li-donor and a deep Na-related acceptor. From ENDOR experiments it is concluded that these deep acceptors are located at the $\mathrm{ZnO} / \mathrm{Zn}(\mathrm{OH})_{2}$ interface.

The saturation of the EPR transition of the shallow donors in $\mathrm{ZnO}$ single crystals at high frequency and low temperature leads to an almost complete polarization of the ${ }^{67} \mathrm{Zn}(I=5 / 2)$ nuclear spins [20]. During the EPR experiments on the SDs in $\mathrm{ZnO}$ quantum dots it was observed that prolonged irradiation of the EPR transition of this donor produces a hole in the EPR resonance line. It was shown [9] that this hole burning is caused by an almost complete polarization of the ${ }^{67} \mathrm{Zn}$ nuclear spins in $\mathrm{ZnO}$ core and of ${ }^{1} \mathrm{H}(I=1 / 2)$ spins in the $\mathrm{Zn}(\mathrm{OH})_{2}$ capping layer.

\section{Experimental}

The preparation of the samples of free-standing hydroxyl-capped $\mathrm{ZnO}$ nanocrystals in the form of dry powders was achieved using a modified version of methods reported in the literature [21-24]. Our method is based on the hydrolysis of $\mathrm{Zn}^{2+}$ ions in absolute alcohols (ethanol or 1-butanol), using either $\mathrm{LiOH} \cdot \mathrm{H}_{2} \mathrm{O}$ for the Li-doped nanocrystals [3, 5] or $\mathrm{NaOH}$ for the Na-doped nanocrystals [7]. Samples of $\mathrm{ZnO}$ doped with $\mathrm{Al}$ were prepared in a similar way [8] by carrying out the hydrolysis of the $\mathrm{Zn}^{2+}$ ions (from $\mathrm{Zn}(\mathrm{Ac})_{2} \cdot 2 \mathrm{H}_{2} \mathrm{O}$ ) in the presence of $\mathrm{Al}^{3+}$ ions $\left(0.5 \mathrm{~mol} \% \mathrm{Al}\left(\mathrm{NO}_{3}\right)_{2} \cdot 9 \mathrm{H}_{2} \mathrm{O}\right.$ in ethanol solution) [8]. The size of the nanocrystals was controlled by the growth duration. The average diameter of the nanocrystals was estimated by X-ray powder diffraction, based on the peak broadening due to the finite crystallite sizes (Scherrer's equation), and by ultraviolet (UV)-visible absorption spectroscopy, based on the size dependence of the band gap owing to quantum confinement effects and using a calibration curve [21, 22]. Free-standing $\mathrm{ZnO}$ nanocrystals with different diameters of 2.2, 2.34, 2.8, 3.4, 4.0, 4.2, 5.6, and $6 \mathrm{~nm}$ were prepared. The surface of the as-prepared $\mathrm{ZnO}$ dots is capped by a thin layer (about one monolayer) of $\mathrm{Zn}(\mathrm{OH})_{2}$ and thus the dots consist of a $\mathrm{ZnO}$ / $\mathrm{Zn}(\mathrm{OH})_{2}$ core-shell structure. Free-standing polyphosphate-capped CdS nanocrystals with diameters of 2 and $3.3 \mathrm{~nm}$ were prepared using a modified version of the 
method described in Ref. [25] by replacing the $\mathrm{Zn}$ salts by $\mathrm{Cd}$ salts. The nanocrystals were illuminated with UV above-band-gap light to create paramagnetic electron and hole centers.

The EPR and ENDOR experiments were performed at temperatures ranging from 1.2 to $10 \mathrm{~K}$ using pulsed EPR spectrometers operating at $95 \mathrm{GHz}$ [26] and $275 \mathrm{GHz}$ [27]. The great advantage of working at this high microwave frequency is that a high spectral resolution is obtained in the EPR as well as in the ENDOR spectra. In addition the use of pulsed microwave techniques facilitates considerably the observation of the ENDOR spectra. The EPR spectra were recorded by monitoring the electron spin echo (ESE) signal following a microwave $\pi / 2$ - and a $\pi$-pulse sequence as a function of the magnetic field. The ENDOR spectra were obtained by monitoring the intensity of the stimulated echo, following three microwave $\pi / 2$-pulses, as a function of the frequency of a radio-frequency pulse applied between the second and the third microwave pulses (Mims-type stimulated-echo pulse sequence) [28].

\section{Results}

\subsection{The Identification of the Binding Core of Shallow Donors in ZnO Quantum Dots}

Figure 1 shows the ESE-detected EPR spectrum of a dry powder sample of Lidoped $\mathrm{ZnO}$ QDs with an average diameter of $3.4 \mathrm{~nm}$ [3]. The EPR spectrum appears after illumination with above-band-gap light during $30 \mathrm{~min}$ at $1.6 \mathrm{~K}$ and persists at low temperature after switching off the light. The spectrum disappears when the temperature is increased above $200 \mathrm{~K}$. The signal labeled (I) at $3.4600 \mathrm{~T}$ with a line width of $6.0 \mathrm{mT}$ is assigned to the shallow donor. Its average $g$-value $g_{\text {av }}=1.9666$ differs somewhat from the $g_{\|}=1.9569$ and $g_{\perp}=1.9552$ values obtained for the interstitial-hydrogen donor in a single crystal of $\mathrm{ZnO}$ [9]. The line width, however, corresponds very well with the value $g_{\|}-g_{\perp}=0.0017$ obtained for the shallow donor in a $\mathrm{ZnO}$ single crystal and taking into account the random character of the powder sample. The result of averaging of the signal, assuming that the $\mathrm{ZnO}$ nanocrystals are randomly oriented, gives rise to a line width of about $3 \mathrm{mT}$ which is slightly smaller than that of the shallow donor signal observed in $\mathrm{ZnO}$ nanocrystals. The size dispersion of the $\mathrm{ZnO}$ nanocrystals of about $5 \%$ is probably responsible for the additional broadening of the line. The EPR signals labeled (II) and (III) are assigned to deep acceptors [3]. Transmission electron microscopy (TEM) image of $\mathrm{ZnO}$ QDs and a model of the $\mathrm{ZnO} / \mathrm{Zn}(\mathrm{OH})_{2}$ core-shell structure are shown in the top of Fig. 1.

First, we will concentrate on the EPR signal (I) assigned to the shallow donor. The EPR signal (I) in Fig. 1 is assigned to a donor because $g_{\text {av }}$ is smaller than the $g$-value of a free electron. The shallow character becomes clear from the dependence of $g_{\text {av }}$ on the size of the QDs as we will discuss in the next section. This shift towards the free-electron $g_{\mathrm{e}}$-value with decrease of QD size is caused by the confinement of the hydrogen-like $1 s$-type wave function of shallow donors when the Bohr radius becomes comparable to the size of the QD. 


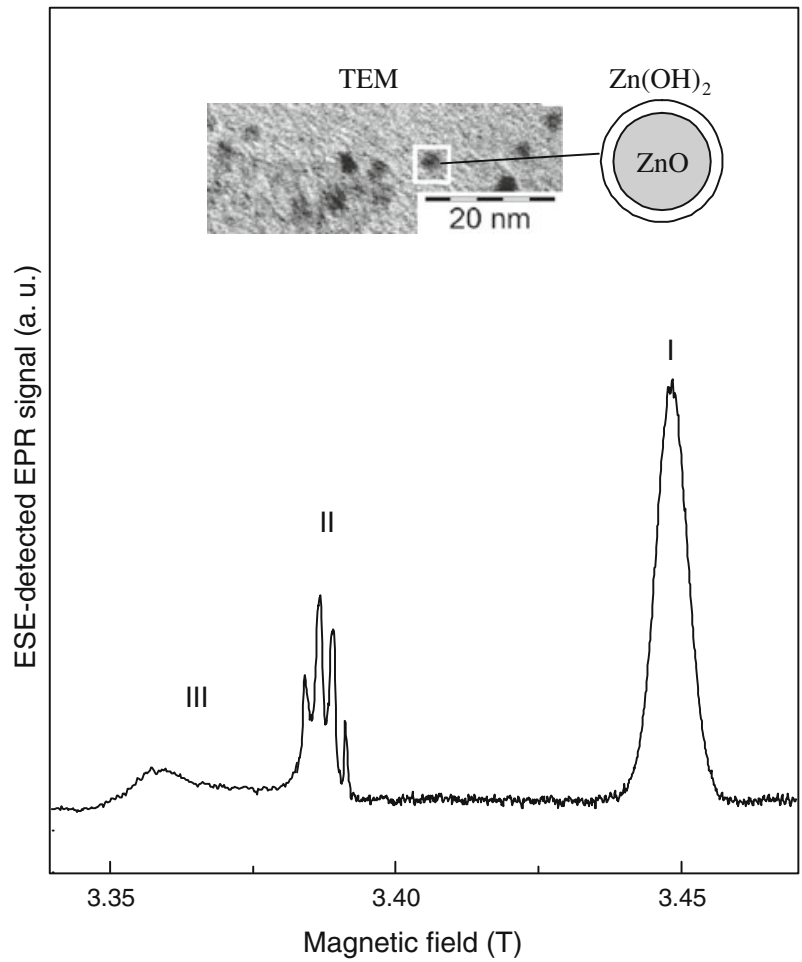

Fig. 1 ESE-detected EPR spectrum at $94.9 \mathrm{GHz}$ and $T=1.5 \mathrm{~K}$ of a dry powder sample of Li-doped $\mathrm{ZnO}$ quantum dots with an average diameter of $3.4 \mathrm{~nm}$ after $30 \mathrm{~min}$ UV irradiation. TEM image of $\mathrm{ZnO}$ nanoparticles $(l e f t)$ and a model of the $\mathrm{ZnO} / \mathrm{Zn}(\mathrm{OH})_{2}$ core-shell structure (right) are shown in the top part of the figure

The EPR spectra in Fig. 1 do not provide information on the chemical nature of the donor since no resolved hyperfine structure is observed, as is the case for the In and Ga shallow donors in $\mathrm{ZnO}$ bulk crystals. To identify the binding core, ENDOR experiments were performed. In Fig. 2 the ENDOR signals are presented as obtained on the EPR signal I of the shallow donor. To understand these results we consider the isotropic hyperfine interaction or Fermi contact term $a_{i}$ which reflects the spin density of the donor electron wave function $(\Psi)$ at the site of the nucleus $\left(r_{i}\right)$

$$
a_{i}=(8 \pi / 3) g_{\mathrm{e}} \beta_{\mathrm{e}} g_{\mathrm{n} i} \beta_{\mathrm{n}}\left|\Psi\left(r_{i}\right)\right|^{2},
$$

where $g_{\mathrm{e}}$ is the electronic $g$-factor, $\beta_{\mathrm{e}}$ is the electronic Bohr magneton, $g_{\mathrm{n} i}$ is the $g$-factor of nucleus $i$, and $\beta_{\mathrm{n}}$ is the nuclear magneton. The related ENDOR transition frequencies are

$$
v_{\mathrm{ENDOR} i}=h^{-1}\left|g_{\mathrm{n} i} \beta_{\mathrm{n}} B_{0} \pm a_{i} / 2\right| .
$$

Equation (2) predicts that each nucleus $i$ gives rise to two ENDOR transitions symmetrically placed around its nuclear Zeeman frequency $g_{\mathrm{n} i} \beta_{\mathrm{n}} B_{0} / h$ when the quadrupole interaction (QI) is neglected and when $a_{i}<g_{\mathrm{n} i} \beta_{\mathrm{n}} B_{0}$. 


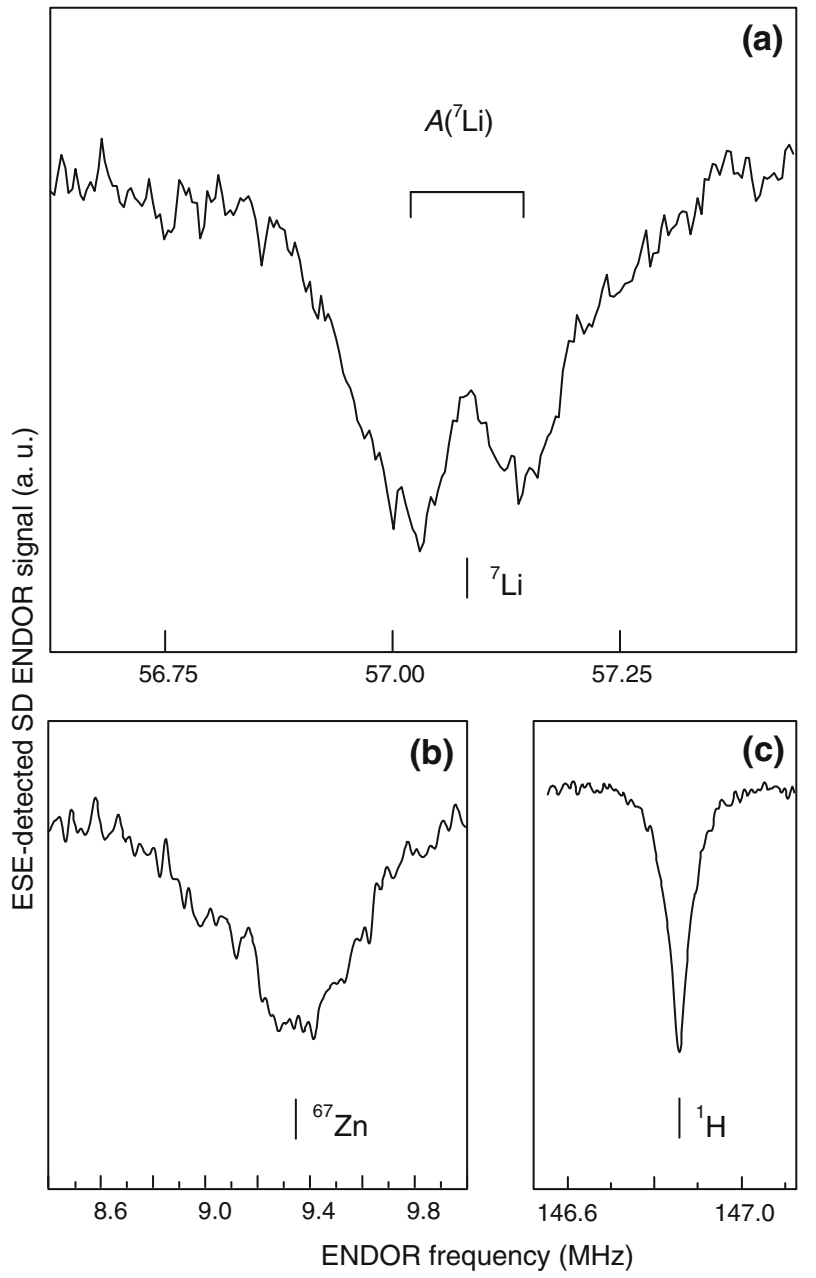

Fig. 2 ESE-detected ENDOR transitions of ${ }^{7} \mathrm{Li}(\mathbf{a}),{ }^{67} \mathrm{Zn}(\mathbf{b})$ and ${ }^{1} \mathrm{H}(\mathbf{c})$ nuclear spins as observed in the EPR signal I (see Fig. 1) of the shallow donors in Li-doped $\mathrm{ZnO}$ quantum dots with an average size of $3.4 \mathrm{~nm}$ recorded at $94.9 \mathrm{GHz}$ and $T=1.8 \mathrm{~K}$. The two ENDOR transitions are symmetrically placed around the Zeeman frequency of ${ }^{7} \mathrm{Li}$ (marked by a bar)

As seen in Fig. 2a, symmetrically around the Zeeman frequency of ${ }^{7} \mathrm{Li}(I=3 / 2$, abundance $92.5 \%$ ) at $57.1 \mathrm{MHz}$ two ENDOR lines separated by $90 \mathrm{kHz}$ are present, which are assigned to ${ }^{7} \mathrm{Li}$. The observation of the ENDOR transitions of the ${ }^{7} \mathrm{Li}$ nuclear spins in the EPR signal of the shallow donor gives an unambiguous identification of the shallow donor as a Li-related center [3]. Moreover, the observed HF splitting gives direct information about the density of unpaired electron spin of the SD at the Li nucleus since the isotropic HF splitting is proportional to the wavefunction density. Our finding confirms the results of Park et al. [29], who predicted on the basis of theoretical calculations that $\mathrm{Li}$ and $\mathrm{Na}$ at interstitial sites in $\mathrm{ZnO}$ behave as shallow donors. Apparently, $\mathrm{Li}^{+}$forms an interstitial core for the shallow 
donor electron in the $\mathrm{ZnO}$ nanocrystal, similar to hydrogen in $\mathrm{ZnO}$ single crystals [18]. Further, it is seen in Fig. 2b that symmetrically around the Zeeman frequency of ${ }^{67} \mathrm{Zn}(I=5 / 2$, abundance $4.1 \%)$ at $9.2 \mathrm{MHz}$ a broad, unresolved set of ENDOR lines of ${ }^{67} \mathrm{Zn}$ spins is present. From the multitude of lines it is clear that we are indeed dealing with a delocalized electron of a shallow donor that interacts with a large number (tens) of ${ }^{67} \mathrm{Zn}$ nuclei [3].

Figure 2c shows an ENDOR line with a width $\Delta v=60 \mathrm{kHz}$ present exactly at the Zeeman frequency of ${ }^{1} \mathrm{H}$. From the width we deduce a ${ }^{1} \mathrm{H}$ HF interaction smaller than $60 \mathrm{kHz}$. This should be compared to our previous observation on the hydrogenrelated shallow donor in a bulk crystal of $\mathrm{ZnO}$ where two ENDOR lines were found with a HF splitting of $1.4 \mathrm{MHz}$ [18]. We conclude that the observed ENDOR lines originate in the hydrogen atoms present in the $\mathrm{Zn}(\mathrm{OH})_{2}$ capping layer where the density of the electronic wave function is very small [3].

A sketch of the hydrogen-like wave function in $\mathrm{ZnO}$ QDs together with a model of the $\mathrm{ZnO} / \mathrm{Zn}(\mathrm{OH})_{2}$ core-shell structure are shown in Fig. 3.

To check whether interstitial $\mathrm{Na}$ can also act as a shallow donor in $\mathrm{ZnO}$, we have performed similar EPR and ENDOR experiments on ZnO QDs that were prepared using $\mathrm{NaOH}$ instead of $\mathrm{LiOH}$ as catalyst. In such $\mathrm{ZnO}$ QDs with a diameter of $3.0 \mathrm{~nm}$, we observe again three EPR signals in analogy to the Li-doped sample. The

Fig. 3 Sketch of the wave function of the shallow donor in quantum dot and a model of the $\mathrm{ZnO} / \mathrm{Zn}(\mathrm{OH})_{2}$ core-shell structure
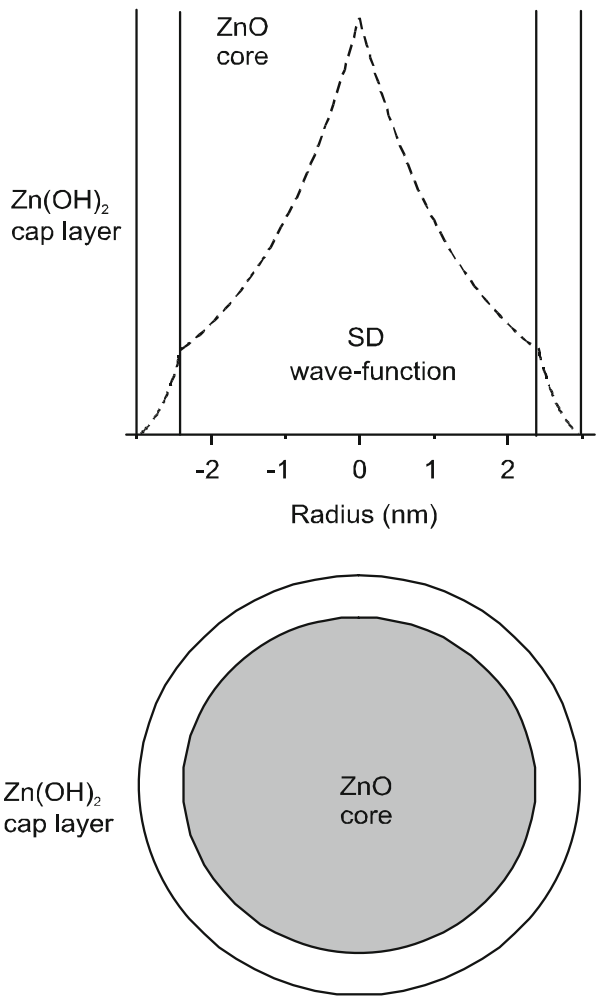
EPR signal similar to (I) in Fig. 1 , with a $g_{\text {av }}=1.9592$, is assigned to a shallow Na-related donor. Figure 4 shows the result of an ENDOR experiment on this signal that reveals two transitions with a splitting of $300 \mathrm{kHz}$ symmetrically placed around the Zeeman frequency of ${ }^{23} \mathrm{Na}$ at $38.97 \mathrm{MHz}$. We consider this as a proof of the presence of a shallow donor related to interstitial $\mathrm{Na}$ in the $\mathrm{ZnO}$ nanocrystal.

Figure 5 shows the ENDOR spectrum of ${ }^{27} \mathrm{Al}$ nuclei as observed in the EPR signal of the shallow donor in $\mathrm{ZnO}$ :Al nanocrystals with a radius of about $2.8 \mathrm{~nm}$ for two values of the magnetic field: $B_{0}=3,459 \mathrm{mT}$ and $B_{0}=3,460 \mathrm{mT}$. The shape of the ENDOR spectrum of the ${ }^{27} \mathrm{Al}$ nuclear spins as observed in the $\mathrm{ZnO}$ nanocrystals is caused by the distribution of quadrupole and HF interactions. The ENDOR spectrum is a result of averaging of these interactions in the randomly oriented $\mathrm{ZnO}$ nanocrystals. It is seen that symmetrically around the nuclear Zeeman frequency of ${ }^{27} \mathrm{Al}(I=5 / 2$, abundance $100 \%)$ at $38.4 \mathrm{MHz}$ two broad ENDOR lines separated by $1.45 \mathrm{MHz}$ are present, which are assigned to HF interaction with the ${ }^{27} \mathrm{Al}$ nucleus. This splitting corresponds to a ${ }^{27} \mathrm{Al}$ hyperfine interaction constant $A\left({ }^{27} \mathrm{Al}\right)=1.45 \mathrm{MHz}$. For $B_{0}=3,460 \mathrm{mT}$ a box-like distribution of ENDOR lines is observed. Since the EPR spectrum of the shallow donors is anisotropic, this field selects mainly a set of nanocrystals with their $c$-axes perpendicular to the magnetic field. The box-like form of the ENDOR spectrum is explained by the quadrupole interaction of the ${ }^{27} \mathrm{Al}$ nuclei that gives rise to five unresolved ENDOR lines.

To account for the quadrupole interaction in the case of axial symmetry the term $h^{-1} m_{q} 3 q_{i}\left(3 \cos ^{2} \theta-1\right)$ must be added to the equation of $v_{\text {ENDOR }}$, where $m_{q}$ is the average

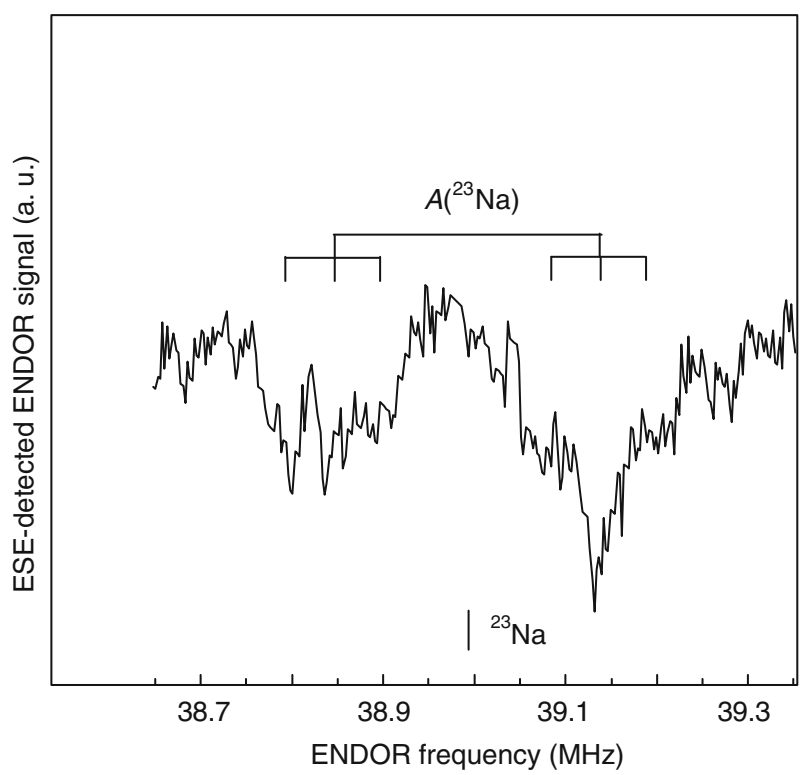

Fig. 4 ESE-detected ENDOR transitions of the ${ }^{23} \mathrm{Na}$ nuclear spins as observed in the EPR signal of the shallow donor in Na-doped $\mathrm{ZnO}$ quantum dots with an average size of $3.0 \mathrm{~nm}$ recorded at $94.9 \mathrm{GHz}$ and $T=1.8 \mathrm{~K}$. The two ENDOR transitions are symmetrically placed around the Zeeman frequency of ${ }^{23} \mathrm{Na}$ (marked by a bar) 


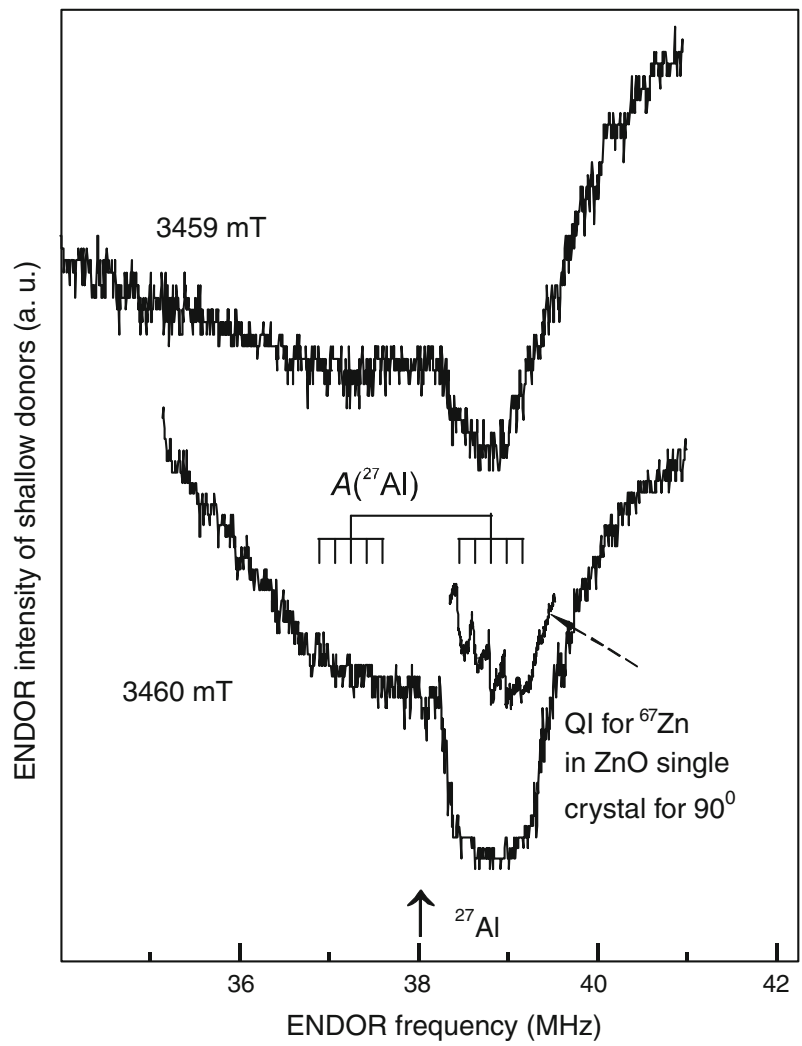

Fig. 5 ESE-detected ENDOR signal of the ${ }^{27} \mathrm{Al}$ nucleus observed in the EPR signal of the shallow donor in $\mathrm{ZnO}: \mathrm{Al}$ quantum dots with a radius of about $2.8 \mathrm{~nm}$ recorded at $94.9 \mathrm{GHz}$ and $T=2 \mathrm{~K}$ for two values of the magnetic field: $B_{0}=3,459 \mathrm{mT}$ and $B_{0}=3,460 \mathrm{mT}$. A part of the ENDOR signal of the ${ }^{67} \mathrm{Zn}$ nucleus observed in the EPR signal of the shallow donor in $\mathrm{ZnO}$ single crystal with resolved QI at $B \perp c$ is given for comparison

value of the two nuclear quantum states $m_{I}$ and $m_{I}$, between which the nuclear transition takes place. For axial symmetry, one has $q=\left(e Q_{0}\right) /[4 I(2 I-1)] V_{Z Z}\left(r_{i}\right)$ where $Q_{0}$ is the electric quadrupole moment in multiples of $|e| \times 10^{-24} \mathrm{~cm}^{2}$ and $V_{\mathrm{ZZ}}\left(r_{i}\right)$ is the electric-field gradient. For ${ }^{27} \mathrm{Al}$ nuclei, the nuclear spin $I=5 / 2$ and $Q_{0}\left({ }^{27} \mathrm{Al}\right)=0.150$. For $I=5 / 2$, there are five $m_{q}$-values: $m_{q}= \pm 2, \pm 1$ and 0 . Thus, the quintet character of the ENDOR spectrum should be observed for Al nucleus, but the quadrupole splitting is not resolved in Fig. 5.

It is important to note that the value of the quadrupole interaction of ${ }^{27} \mathrm{Al}$ is almost equal to that of the ${ }^{67} \mathrm{Zn}$ nuclear spins in $\mathrm{ZnO}$. First, the values of the quadrupole moments and, second, the nuclear spins of ${ }^{27} \mathrm{Al}$ and ${ }^{67} \mathrm{Zn}$ are the same. For comparison, the ENDOR spectrum of the ${ }^{67} \mathrm{Zn}$ nuclei observed for the shallow H-related donor in $\mathrm{ZnO}$ single crystals with $B \perp c$ is presented in Fig. 5 on the same scale as the ENDOR signals of ${ }^{27} \mathrm{Al}$. This shows that the intrinsic electric-field gradients at the $\mathrm{Zn}$ nuclear sites and $\mathrm{Al}$ site are virtually the same. This finding is taken as a proof that $\mathrm{Al}$ enters substitutionally in the $\mathrm{ZnO}$ nanocrystals and it is 
centrally located at a $\mathrm{Zn}$ position and forms a core for the shallow donor electron in the $\mathrm{ZnO}$ nanoparticle. This is not obvious because the small radius of $\mathrm{Al}^{3+}(0.51 \AA$ compared to $0.74 \AA$ for $\mathrm{Zn}^{2+}$ ) could drive the impurity into an off-center position.

In general, quadrupole interactions should also be observed in the ENDOR spectra presented in Figs. 2 and 4 for Li- and Na-related SDs since ${ }^{7} \mathrm{Li}$ and ${ }^{23} \mathrm{Na}$ have nuclear spins $I=3 / 2$ and quadrupole moments $Q_{0}\left({ }^{7} \mathrm{Li}\right)=-0.040$ and $Q_{0}\left({ }^{23} \mathrm{Na}\right)=0.103$. However, the quadrupole splitting is not resolved and could only be estimated from the line width. The pattern with the assumed quadrupole splitting for the Na-related shallow donors is presented in Fig. 4. Our estimations, which are more reliable for Na-related SDs (larger quadrupole moment), show that the electric-field gradients at the $\mathrm{Li}$ and $\mathrm{Na}$ nuclear sites are about two times smaller than the intrinsic electric-field gradient at the $\mathrm{Zn}$ site. This is in line with the suggestion that $\mathrm{Li}$ and $\mathrm{Na}$ occupy an interstitional position to create the shallow donor.

\subsection{Probing the Wave Function of Shallow Donors in ZnO Quantum Dots and Confinement Effects}

The ENDOR studies allow us to probe the effects of confinement on the spatially extended wave function of the shallow donor by measuring the isotropic HF interaction, which reflects the spin density at the site of the nucleus, and by varying the particle size in the quantum-size regime. The ${ }^{7} \mathrm{Li}$ ENDOR signals are excellently suited for this purpose and thus we have measured the dependence of the splitting of the two ${ }^{7} \mathrm{Li}$ hyperfine components on the radius $R$ of the $\mathrm{ZnO}$ core of the QDs. Figure 6a shows the values of the isotropic HF interaction $A$ of the ${ }^{7} \mathrm{Li}$ nuclear spin of the shallow Li-related donor in $\mathrm{ZnO}$ QDs with radii between 3.0 and $1.1 \mathrm{~nm}$ as observed in the ENDOR spectra at $T=1.2 \mathrm{~K}$. The error bar in the values of $A$ is estimated from the noise in the ENDOR spectra. The variation in the size of the particles is derived from TEM and X-ray diffraction (XRD) measurements. The dashed line in Fig. 6 is a fit to the measured values of $A$ for QDs with radii between 3.0 and $1.5 \mathrm{~nm}$ using the function $\sim r_{\mathrm{QD}}^{-3}$. Down to $R=1.5 \mathrm{~nm}$, a value which is equal to the Bohr radius of the shallow donor, the experimental results follow quite closely this dependence, while for smaller radii there is a considerable deviation [5].

In addition to the hyperfine interactions with the ${ }^{7} \mathrm{Li}$ nucleus, information can be also obtained from the hyperfine interactions with the ${ }^{67} \mathrm{Zn}$ nuclei. In Fig. 7 the ENDOR spectra of the ${ }^{67} \mathrm{Zn}$ nuclei are presented (symmetrically placed around the ${ }^{67} \mathrm{Zn}$ Zeeman frequency at about 9.2 MHz) for $\mathrm{ZnO}$ QDs of various radii: 3.0, 1.6 and $1.17 \mathrm{~nm}$. For comparison, in the lower part of Fig. 7, the ENDOR signals of ${ }^{67} \mathrm{Zn}$ nuclei are presented as observed in the EPR signal of the H-related shallow donor in $\mathrm{ZnO}$ single crystals for three orientations of the magnetic field $\left[0^{\circ}(B \| c)\right.$, $54^{\circ}$ and $90^{\circ}(B \perp c)$ ]. This spectrum reveals the QI for remote $\mathrm{Zn}$ shells. In addition, the high-frequency part of the ENDOR spectrum for $B \| c$ is shown with a higher resolution. It is seen that this spectrum consists of a multitude of ENDOR lines that are related to various Zn shells. The shape of the ENDOR spectrum of the ${ }^{67} \mathrm{Zn}$ nuclear spins as observed in the $\mathrm{ZnO}$ QDs is caused by the distribution of 
Fig. 6 a Isotropic hyperfine interaction $A$ of the ${ }^{7} \mathrm{Li}$ nuclear spin of the shallow Li-related donors in $\mathrm{ZnO}$ quantum dots with radii between 3.0 and $1.1 \mathrm{~nm}$. The black circles indicate the hyperfine splitting as observed in the ENDOR spectra at $T=1.2 \mathrm{~K}$. The error bar in the values of $A_{\text {iso }}$ is estimated from the noise in the ENDOR spectra. The variation in the size of the particles is derived from TEM and XRD measurements. The dashed line is a fit to the measured values of $A$ for the QDs with radii between 3.0 and $1.5 \mathrm{~nm}$ using the function $\sim R^{-3}$. b Variation of the wave function density at the interface of the $\mathrm{ZnO}$ core and the $\mathrm{Zn}(\mathrm{OH})_{2}$ capping layer for the QDs as calculated from the dip in ENDOR of the ${ }^{7} \mathrm{Li}$ nuclear spin (core side, black square) and from the width of the ENDOR line of the ${ }^{1} \mathrm{H}$ nuclear spins in the $\mathrm{Zn}(\mathrm{OH})_{2}$ capping layer (open triangle). The dashed line is a fit to the measured values for the QDs with radii between 3.0 and $1.5 \mathrm{~nm}$ using the function $\sim R^{-3}$

quadrupole and HF interactions inside the nanocrystal. The broad line is a result of averaging of these interactions in the randomly oriented $\mathrm{ZnO}$ nanocrystals.

The remarkable observations in the ENDOR spectra in Fig. 7 are that the distribution of ENDOR lines broadens upon reduction of the size of the QDs and that a dip develops around the Zeeman frequency of the ${ }^{67} \mathrm{Zn}$ nuclear spins. The dip becomes more prominent and broader when the radius of the $\mathrm{ZnO}$ core is reduced from 3.0 to $1.6-1.17 \mathrm{~nm}$. The broadening of the ENDOR band indicates that the maximum density of the electronic wave function increases when reducing the size of the nanoparticles. The disappearance of the ENDOR signals close to the ${ }^{67} \mathrm{Zn}$ Zeeman frequency shows that remote shells are missing in the QD. The dip in the ENDOR spectrum indicates that for the small QDs, the Zn nuclei at the interface carry a nonzero spin density. This conclusion is in line with the observation that the electronic density at the $\mathrm{ZnO} / \mathrm{Zn}(\mathrm{OH})_{2}$ interface, as measured from the line width of the ${ }^{1} \mathrm{H}$ ENDOR signal, increases with decreasing size of the QDs [5]. This width is taken as a measure of the distribution of the hyperfine fields in the capping layer.

The estimates of the electronic density at the $\mathrm{ZnO} / \mathrm{Zn}(\mathrm{OH})_{2}$ interface, using either the width of the dip in the ${ }^{67} \mathrm{Zn}$ ENDOR signal or the line width of the ${ }^{1} \mathrm{H}$ ENDOR signal, and using the amplification factors for $\mathrm{Zn}$ of 1,500 and for $\mathrm{H}$ of 20 [18], give about the same value, in agreement with the wave-function continuity principle. The variation of the density of the wave function at the interface between the $\mathrm{ZnO}$ core and the $\mathrm{Zn}(\mathrm{OH})_{2}$ capping layer for the QDs with radii between 3 and $1.1 \mathrm{~nm}$ is presented in Fig. 6b. For QDs with radii between 3 and $1.5 \mathrm{~nm}$, the $R^{-3}$ dependence describes the experimental data but for smaller radii, a deviation from this dependence is observed.

To test whether the observed size dependence of the density of the wave function at the ${ }^{7} \mathrm{Li}$ nucleus in the $\mathrm{ZnO}$ core and its distribution in the $\mathrm{Zn}(\mathrm{OH})_{2}$ capping layer can be explained with the effective-mass approximation (EMA), a trial wave function with appropriate boundary conditions to simulate the envelope function of the shallow donor electron has been introduced [5]. By using a variational procedure in which the total energy is calculated numerically and minimized, we have derived the density of the wave function at the center of the $\mathrm{ZnO}$ core, where the Li nucleus is supposed to be located, and at the $\mathrm{ZnO} / \mathrm{Zn}(\mathrm{OH})_{2}$ interface. This simple analytical EMA-based model gives a good account for the envelope function of the shallow donor electron for large nanocrystals, but it does not give a stable solution in the quantum confinement regime, i.e., in semiconductor nanocrystals with radii of the order of or smaller than the Bohr radius in the bulk material. There are two possible 

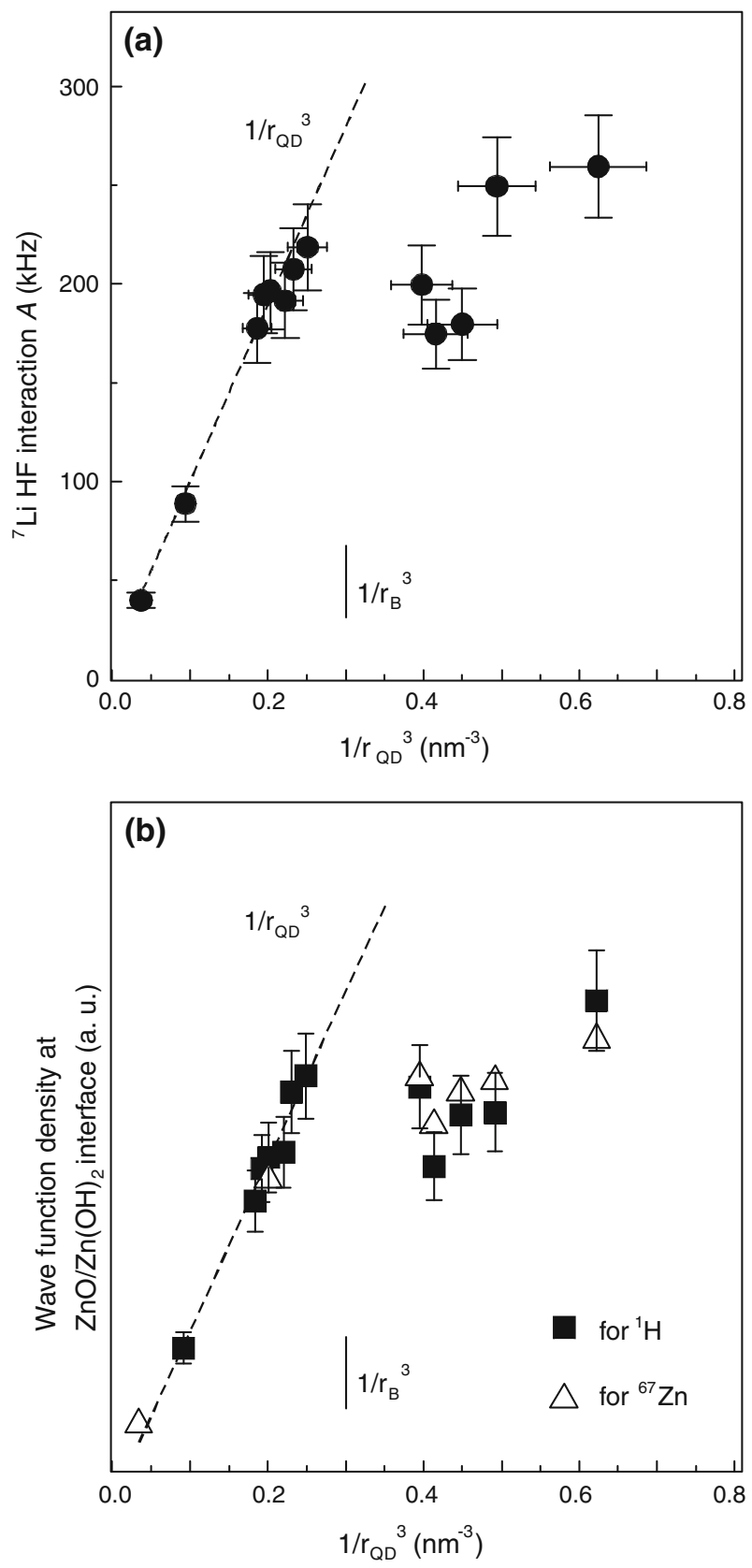

reasons for this failure. First, it is not permitted to use the bulk value for the effective mass of the electron. This parameter reflects the effect of the periodic potential of a (infinite) semiconductor crystal and this approximation breaks down for the nanometer-sized nanocrystal. In other words, the allowed values for the wave 


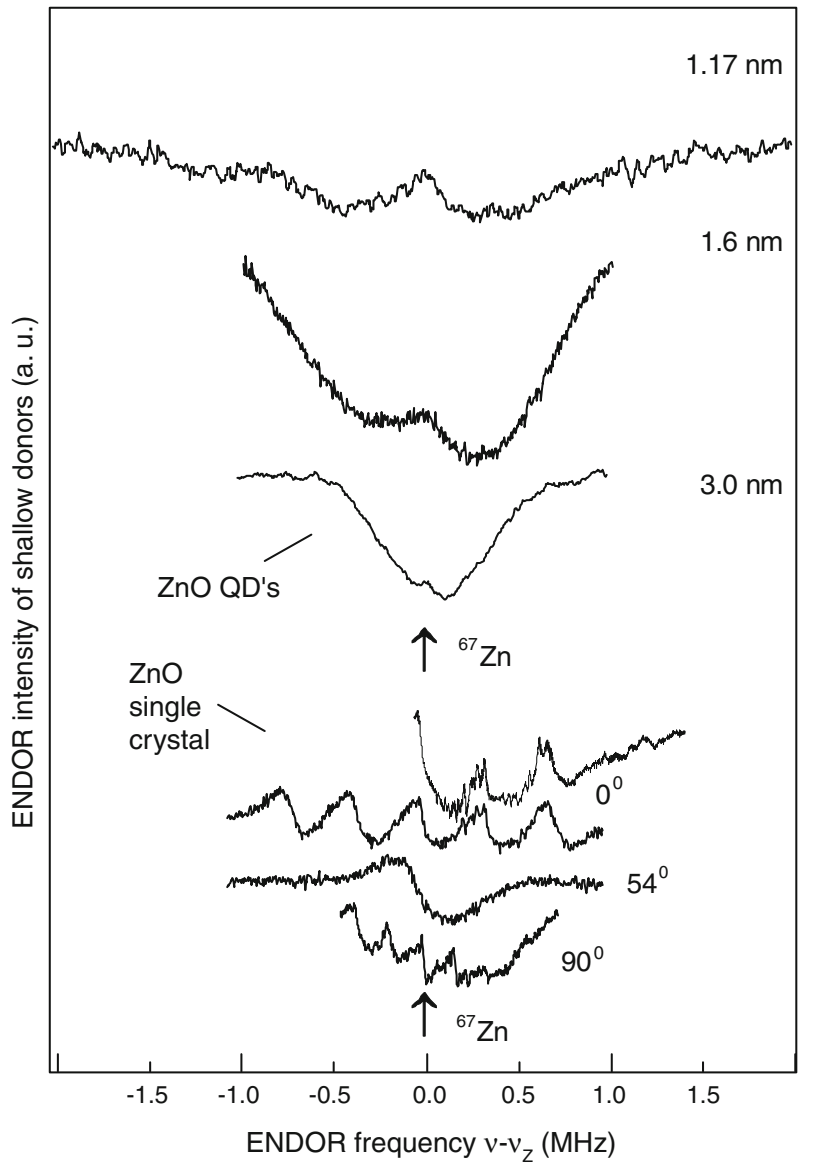

Fig. 7 ENDOR spectra of the ${ }^{67} \mathrm{Zn}$ nuclear spins in the $\mathrm{ZnO}$ quantum dots with radii of 1.17, 1.6 and $3.0 \mathrm{~nm}$ recorded at $94.9 \mathrm{GHz}$ and $T=1.8 \mathrm{~K}$. Each spectrum consists of many unresolved lines placed symmetrically around the Zeeman frequency (marked by arrows) of the ${ }^{67} \mathrm{Zn}$ nuclear spins. In the bottom part the ESE-detected ENDOR signals of ${ }^{67} \mathrm{Zn}$ nuclei observed in the EPR signal of the shallow H-related donors in $\mathrm{ZnO}$ single crystals are presented as measured in three orientations $\left[0^{\circ}(B \| c), 54^{\circ}\right.$ and $90^{\circ}$ $(B \perp c)$ ]. These three recordings reveal the QI of the ${ }^{67} \mathrm{Zn}$ nuclear spins. In addition, the high-frequency part of the ENDOR spectrum with higher resolution is shown for $B \| c$

vector $\mathbf{k}$ become discrete and the energy eigenvalues are those for an electron of mass $m_{0}$ in a box. Secondly, the definition of a dielectric constant as a consequence of the Lorentz relation does not apply to our nanocrystal. As demonstrated in Ref. [30], the effective screening function in a confined system depends on the size of and on the position in the nanoparticle. It is concluded that the EMA-based model does not yield an appropriate description of the electronic wave function when the radius of the QD is reduced below the Bohr radius of the shallow donor. We believe that an appropriate description of the electronic wave function may be found by using molecular-cluster-type calculations as carried out by Melnikov and Chelikowsky [31] for the electronic wave function of shallow P donors in Si nanoparticles. 
The shallow character of the Li-related donor is also visible in the dependence of the $g$-factor on the size of the $\mathrm{ZnO}$ QD as shown in Fig. 8. In this figure we have also incorporated a recording of the EPR signal of the substitutional Al donor in $\mathrm{ZnO}$ nanocrystals. The shift of the $g$-factor towards the free-electron value when reducing the size of the nanoparticles is caused by the confinement of the hydrogenlike $1 s$-type wave function of shallow donors when the Bohr radius becomes comparable to the size of the nanocrystals. The effect is explained by the reduction of the admixture of valence-band states and higher-lying conduction bands into the lowest conduction band by the increase of the band-gap energy and the energy of higher-lying conduction bands upon the reduction of the size of the nanocrystals [32]. For comparison, the ESE-detected EPR spectra of the shallow hydrogenrelated donors in a single crystal of $\mathrm{ZnO}$ are presented in the top of Fig. 8 for several orientations of the magnetic field with respect to the $c$-axis. The frequency, the temperature and the magnetic-field scales are the same for $\mathrm{ZnO}$ QDs and for $\mathrm{ZnO}$ single crystals. Averaging of the signal, on the assumption that the $\mathrm{ZnO}$

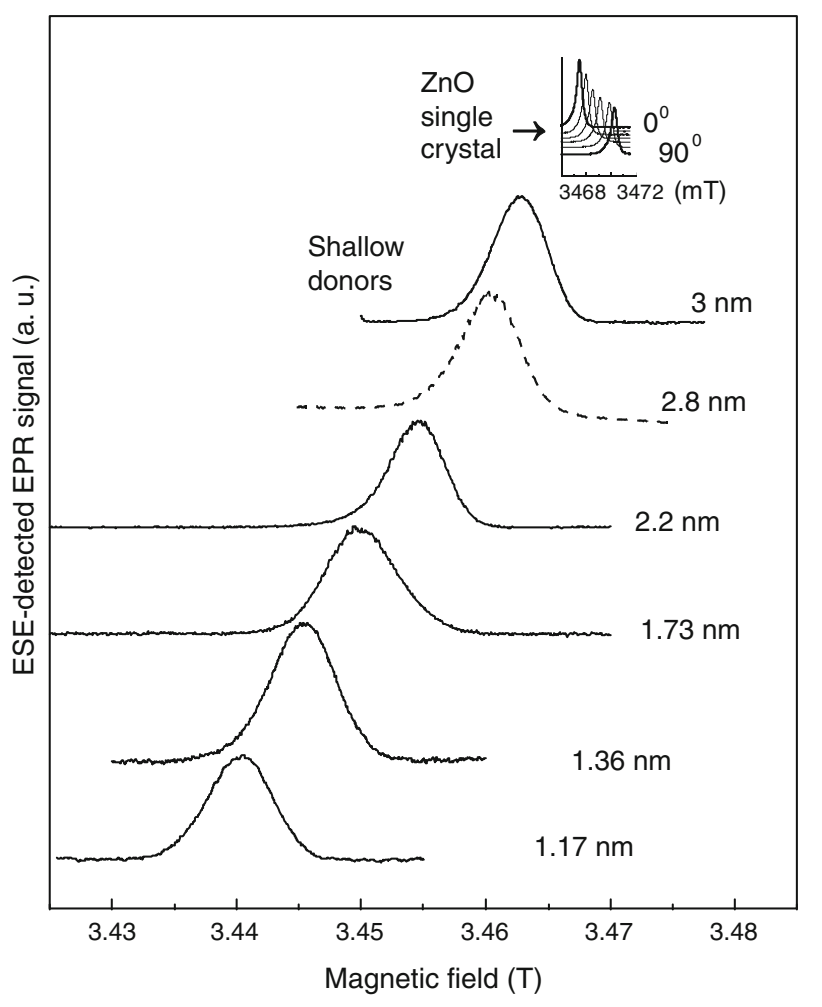

Fig. 8 ESE-detected EPR spectra of SDs in ZnO QDs with diameters of 1.17, 1.36, 1.73, 2.2, 2.8 and $3 \mathrm{~nm}$ recorded at $94.9 \mathrm{GHz}$ and $T=2 \mathrm{~K}$. The inset shows the angular dependence of the ESE-detected EPR spectra of H-related SDs in $\mathrm{ZnO}$ single crystal presented on the same magnetic-field scale. The dashed line is the ESE-detected EPR spectra of ZnO:Al QD's. The solid lines are the ESE-detected EPR spectra of ZnO:Li QD's 
nanocrystals are randomly oriented, gives a line width of about $3 \mathrm{mT}$. The size dispersion of the $\mathrm{ZnO}$ nanocrystals of about $5 \%$ is probably responsible for the additional broadening of the line.

Figure 9a shows the ESE-detected EPR spectrum of SDs in CdS quantum dots with radii of 1.65 and $1 \mathrm{~nm}$ registered after 30-min light excitation at $94.9 \mathrm{GHz}$ and $T=2.0 \mathrm{~K}$. The influence of the confinement effect on the $g$-factor of these SDs is visible in Fig. 9b. In the same figure the dependence of the $g$-factor of the shallow Li-related donor on the size of $\mathrm{ZnO}$ QDs due to the confinement effect is also displayed. One can see that the data on $\mathrm{ZnO}$ and $\mathrm{CdS}$ QDs cannot be fitted with the same dependence. It remains for theoreticians to explain these different behaviors.
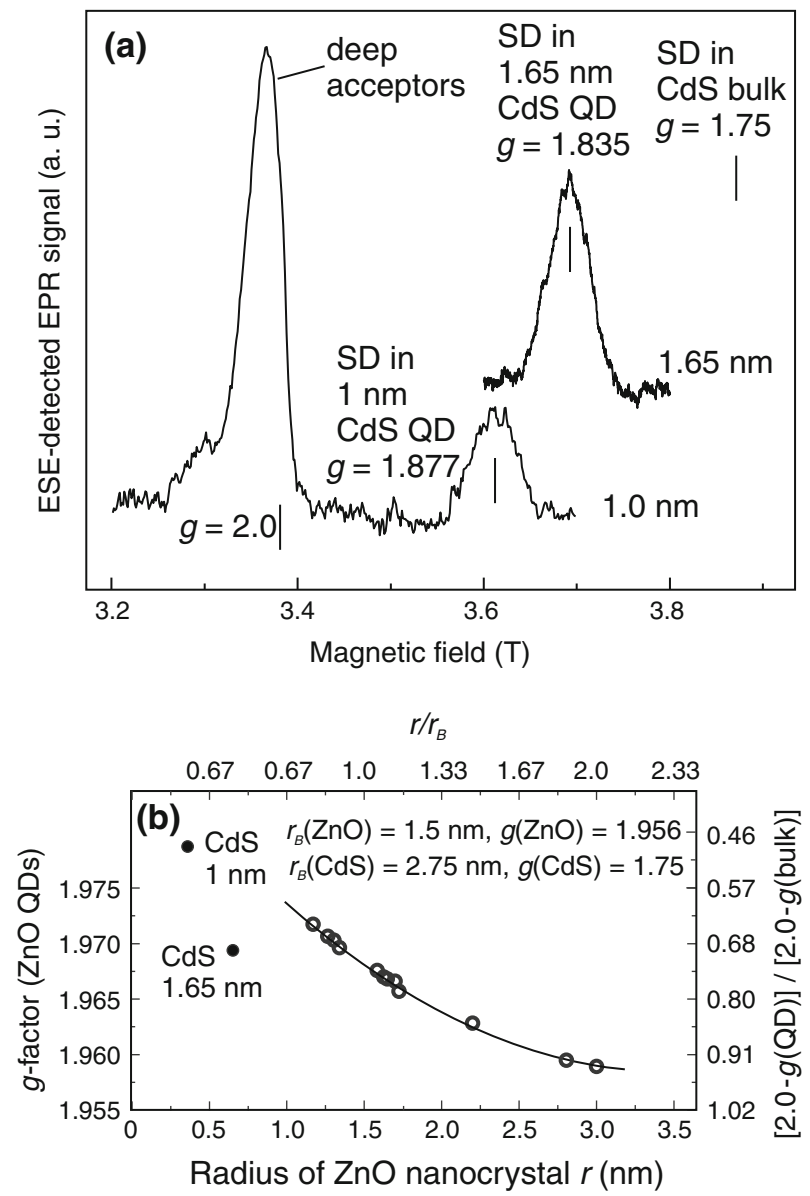

Fig. 9 a ESE-detected EPR spectra of SDs in CdS QDs with radii of 1.65 and $1.0 \mathrm{~nm}$ recorded at 94.9 GHz and $T=2.0 \mathrm{~K}$. b Dependence of the $g$-factor of the shallow donor on the size of ZnO QD. The upper scale gives a QD radius normalized to the Bohr radius $r_{\mathrm{B}}$ of the SD in the related bulk semiconductor. The left scale gives a $g$-factor shift of the SD in QD from $g=2.0$ normalized to that for the bulk material. The filled circle indicates the $g$-factor shift in CdS QDs on the normalized scale 


\subsection{Dynamic Nuclear Polarization (DNP) of Nuclear Spins}

Figure 10a shows the ESE-detected EPR signal of the shallow Li-related donor in a dry powder of $\mathrm{ZnO}$ QDs with a diameter of $4 \mathrm{~nm}$ observed at a frequency of $94.9 \mathrm{GHz}$ and a temperature of $1.5 \mathrm{~K}$. Before the EPR experiment ultraviolet light illuminated the sample at $1.4 \mathrm{~K}$ during $30 \mathrm{~min}$. The upper EPR line (1) in Fig. 10a is recorded without microwave pre-irradiation. The line is slightly asymmetric and its width is caused partly by the anisotropy of the $g$-tensor and partly by the distribution in size of about $10 \%$. The dependence of the $g$-tensor on the size of the nanocrystals is caused by the confinement of the $1 s$-type electronic wave function of the shallow donor that has a Bohr radius comparable to the radius of the nanoparticles. Curve (2) in Fig. 10a is recorded immediately after continuous-wave (cw) microwave pre-irradiation during 3 min with a microwave power of $20 \mathrm{~mW}$ at the center of the unperturbed line (1). It is seen that after the irradiation a hole is burnt in the line and that simultaneously a new sharp peak (an "antihole") appears at the low-field side of the hole. The curve (2-1) represents the difference between curves (2) and (1) and clearly shows the antihole that arises in addition to the hole.

The creation of the hole and antihole in the EPR line is caused by DNP of the ${ }^{67} \mathrm{Zn}(I=5 / 2)$ nuclear spins and, as we will show below, of the ${ }^{1} \mathrm{H}(I=1 / 2)$ nuclear spins in the $\mathrm{Zn}(\mathrm{OH})_{2}$ capping layer. The polarized nuclear spins create an internal magnetic field and, as a result, the resonance line of the electron spins, subjected to the microwave irradiation, shifts to a lower external field value resulting in the hole and the antihole in the inhomogeneously broadened EPR line. In the DNP process of shallow $\mathrm{H}$ donors in a single crystal of $\mathrm{ZnO}$ a similar effect was observed [20]. Since the line width of the $\mathrm{Li}$ donor in the random sample of $\mathrm{ZnO}$ nanocrystals depends to a large extent on the anisotropy of the $g$-tensor, the hole in the line corresponds to electron spins of $\mathrm{Li}$ donors in $\mathrm{ZnO}$ particles with a given orientation of their hexagonal crystal axis with respect to the external magnetic field. The hole decays slowly and disappears in about $30 \mathrm{~min}$. This disappearance is caused by the nuclear spin-lattice relaxation that gradually restores the non-thermal nuclear spin polarization to its equilibrium value.

The striking result is that the intensity of the induced hole depends on the orientation of dry powder sample. The hole was observed to disappear after a rotation by $90^{\circ}$ but after the reverse rotation or a rotation by $180^{\circ}$, the hole reappears at the same position [9]. The attractive feature of this experiment is that one can select a particular orientation of nanoparticles in a random sample.

Figure 10b shows effect of the relaxation of the polarized nuclear spins. This figure shows the EPR signal of the shallow donor in $\mathrm{ZnO}$ nanocrystals with a diameter of $4.2 \mathrm{~nm}$ induced by $3-\mathrm{min} \mathrm{cw}$ microwave pre-irradiation $(20 \mathrm{~mW})$ at $94.9 \mathrm{GHz}$ and $2 \mathrm{~K}$. The upper EPR line in Fig. $10 \mathrm{~b}$ is recorded without preirradiation. The second recording from the top is obtained after $\mathrm{cw}$ microwave irradiation $(20 \mathrm{~mW})$ during $3 \mathrm{~min}$ at the center of the unperturbed line. It is seen that a hole is burned in the center of the line where the irradiation took place. The spectrum labeled by $(0)$ in Fig. 10b represents the difference between two upper curves. Curves labeled (4), (9), (13), (27), (40), and (74) in Fig. 10b are recorded 4, 
Fig. 10 a Effect of $\mathrm{cw}$ resonant microwave irradiation on the line shape of the ESE-detected EPR spectrum of the shallow Li-related donor in $\mathrm{ZnO}$ QDs with a diameter of $4 \mathrm{~nm}$ at $94.9 \mathrm{GHz}$ and $T=2 \mathrm{~K}$. The upper EPR line (1) is recorded without pre-irradiation. The spectrum labeled by (2) is recorded immediately after $20 \mathrm{~mW} \mathrm{cw}$ microwave irradiation during $3 \mathrm{~min}$ at the center of the unperturbed line (1). Curve (2-1) represents the difference between curves (2) and (1). It is seen that a hole is burnt and an antihole develops. b Relaxation of polarization in the EPR signal of the shallow donor in $\mathrm{ZnO}$ nanocrystals with a diameter of $4.2 \mathrm{~nm}$ induced by 3 -min cw microwave irradiation $(20 \mathrm{~mW})$ at $94.9 \mathrm{GHz}$ and $T=2 \mathrm{~K}$. The upper EPR line is recorded without pre-irradiation. The second recording from the top is obtained after $\mathrm{cw}$ microwave irradiation during $3 \mathrm{~min}$ at the center of the unperturbed line. The spectrum labeled by $(0)$ represents the difference between two upper curves. Curves (4), (9), (13), (27), (40), and (74) are recorded 4, 9, 13, 27, 40 and 74 min after the pre-irradiation, respectively (the upper unperturbed line was subtracted from each spectrum). It is seen that slowly the hole and the "antihole" decrease and the EPR spectrum recovers its initial unperturbed form

$9,13,27,40$ and $74 \mathrm{~min}$ after the pre-irradiation, respectively (the upper unperturbed line was subtracted from each spectrum). It is seen that slowly the hole and the "antihole" decrease and the EPR spectrum recovers its initial unperturbed form. One can see that it takes about $75 \mathrm{~min}$ for the hole and the "antihole" to relax back to the original zero value. It should be noted that the relaxation of the polarized nuclear spins back to their equilibrium values strongly depends on the size of the nanocrystals. The relaxation time decreases for smaller QDs, e.g., for the $\mathrm{ZnO}$ nanocrystals with a diameter of $2.8 \mathrm{~nm}$, it takes about $30 \mathrm{~min}$ for the hole in the EPR line to relax back to its original zero value.

In Fig. 11a it is shown how the hole and the antihole shift through the inhomogeneous EPR line of $\mathrm{ZnO}$ QDs with a diameter of $2.8 \mathrm{~nm}$, by exposing the samples to subsequent pre-irradiations with $\mathrm{cw}$ microwaves. The lowest curve in Fig. 11a is recorded without pre-irradiation. The first curve above the lowest one is recorded after $\mathrm{cw}$ microwave irradiation $(20 \mathrm{~mW})$ during $3 \mathrm{~min}$ at the center of the unperturbed line. The next curve is obtained after $\mathrm{cw}$ microwave irradiation during $3 \mathrm{~min}$ at the peak of the antihole. This procedure is repeated and finally the antihole stabilizes at a position shifted by $7 \mathrm{mT}$. In Fig. $11 \mathrm{~b}$ the shift of the hole and the antihole versus the number of 3-min cycles of microwave pre-irradiation is shown for the shallow donor in $\mathrm{ZnO}$ QDs with diameters of 2.8, 3.4, and $4.2 \mathrm{~nm}$ [9]. For comparison, a similar curve is shown for the shift of the EPR line of the shallow donor in a single crystal of $\mathrm{ZnO}$ [20]. It is seen that for particles with an average diameter of $4.2 \mathrm{~nm}$, the maximum shift is about the same as for the single crystal but that the shift increases considerably when performing the experiments on nanocrystals with diameters of 3.4 and $2.8 \mathrm{~nm}$. In the next paragraphs we will present arguments to explain the increase of this shift by DNP of the ${ }^{67} \mathrm{Zn}$ nuclear spins and of the ${ }^{1} \mathrm{H}$ nuclear spins in the $\mathrm{Zn}(\mathrm{OH})_{2}$ capping layer, in combination with the effect of confinement of the electronic wave function of the shallow donor.

To check whether a polarization of the ${ }^{1} \mathrm{H}$ nuclear spins affects the resonance line of the electron spin we have carried out ENDOR experiments on the protons. Curve (1) in Fig. 12a represents again the unperturbed EPR line. Curve (2) is the result obtained after 3 min of microwave irradiation at the peak of the line. Curve (3) is recorded after 3 min of microwave irradiation at the peak of curve 2. In Fig. 12b the ENDOR signals of the ${ }^{1} \mathrm{H}$ nuclear spins detected in curves (1), (2) and (3) of Fig. 12a at the positions indicated by the bar are presented. First of all, it is seen that 

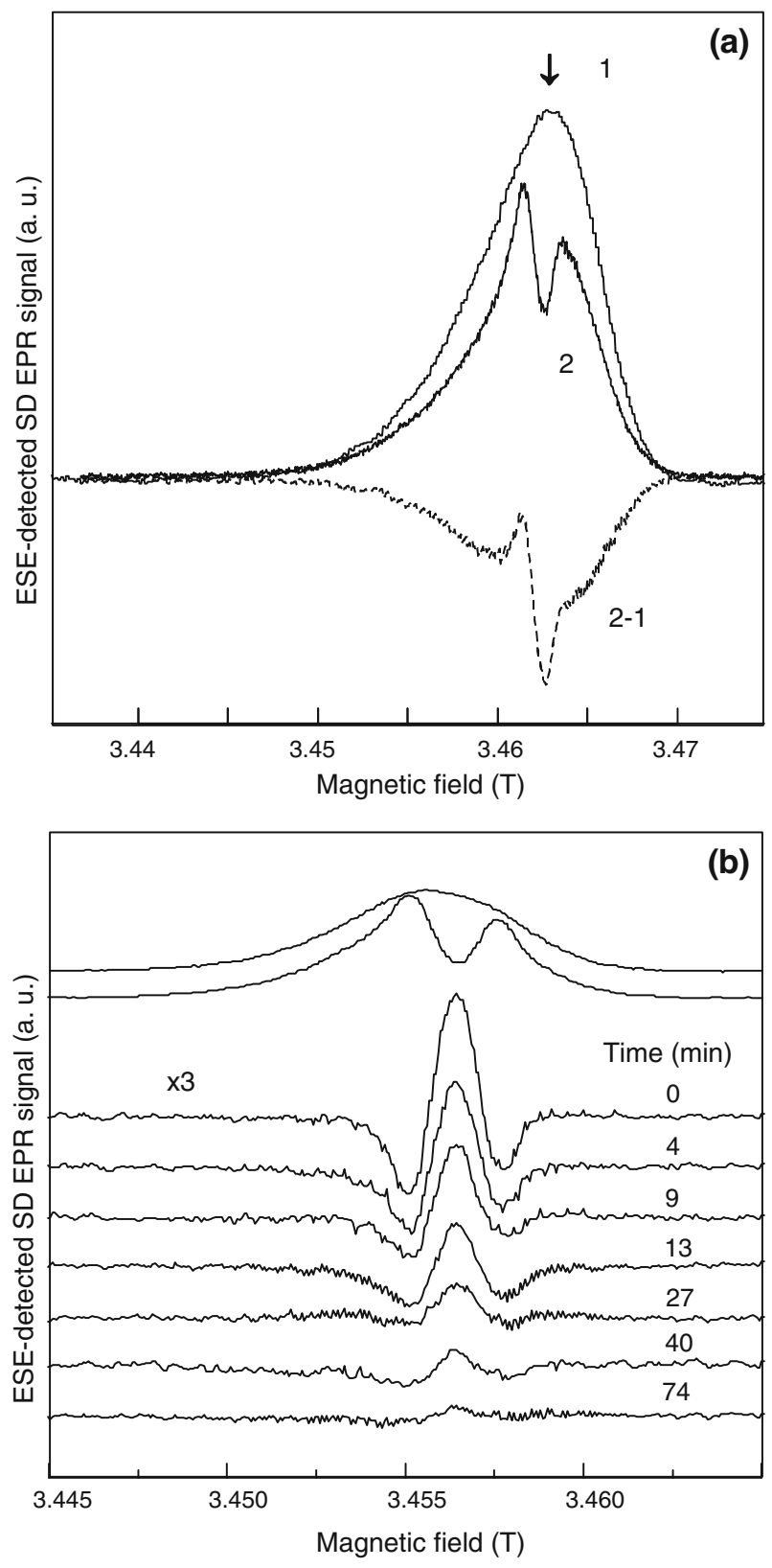

strong ENDOR signals are obtained around the Zeeman frequency of the ${ }^{1} \mathrm{H}$ nuclear spins, indicating that the wave function of the shallow donor extends into the capping layer. Second, the resonance frequency of the ${ }^{1} \mathrm{H}$ nuclear spins shifts to the higher frequency when the magnetic field at which the ENDOR experiment is carried out moves to the lower field values. We explain this behavior by the 
polarization of the ${ }^{1} \mathrm{H}$ nuclear spins, which produces an internal field and thus shifts their resonance frequency to the higher values.

The experiments presented above demonstrate that the ${ }^{67} \mathrm{Zn}$ as well as the ${ }^{1} \mathrm{H}$ nuclear spins become polarized when saturating the EPR transition of the shallow donor in the $\mathrm{ZnO}$ QDs. The dynamic polarization of the ${ }^{67} \mathrm{Zn}$ nuclear spins shows the same behavior as observed for the ${ }^{67} \mathrm{Zn}$ spins in a single crystal of $\mathrm{ZnO}$ doped
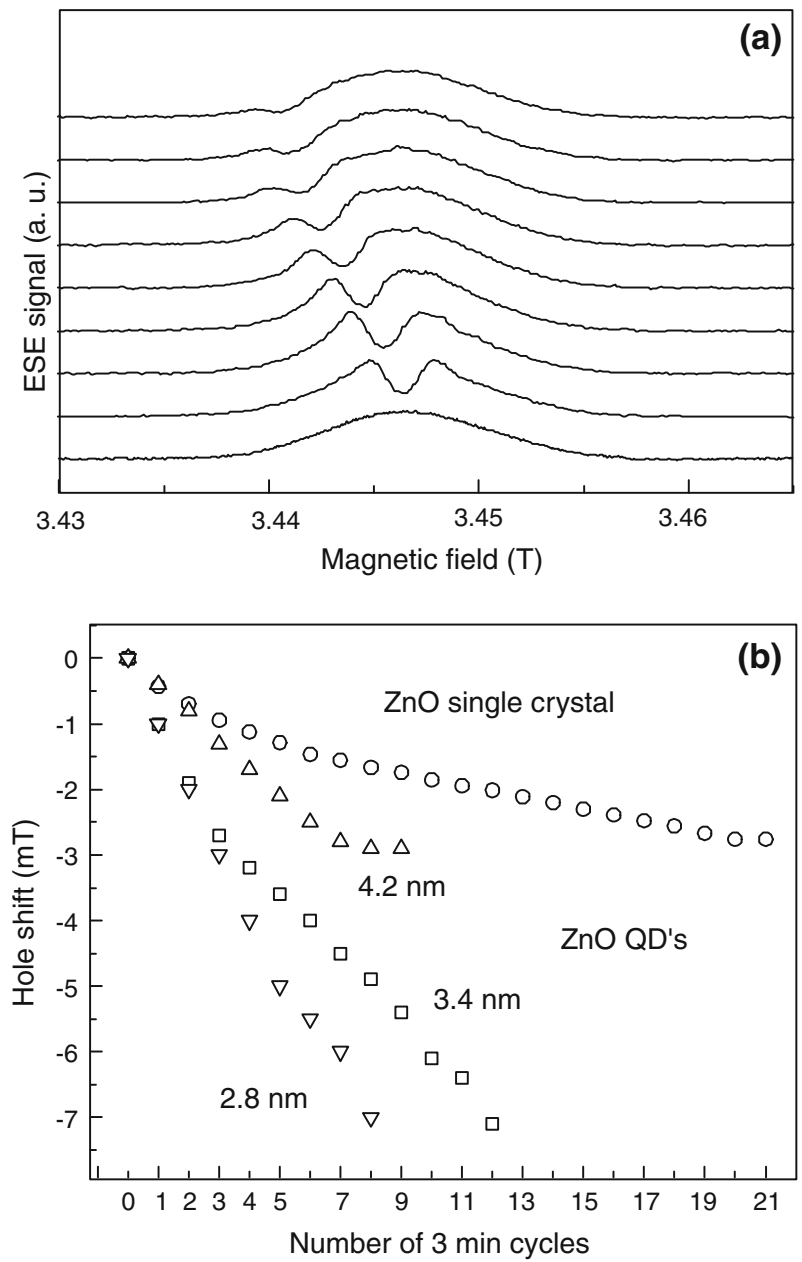

Fig. 11 a Shift of the hole in the EPR transition of the shallow Li donor in ZnO QDs with a diameter of $2.8 \mathrm{~nm}$ induced by cw microwave irradiation at $94.9 \mathrm{GHz}$ and $T=2 \mathrm{~K}$. The lowest $\mathrm{EPR}$ line is recorded without pre-irradiation. The second recording from the bottom is obtained after $\mathrm{cw}$ microwave irradiation during $3 \mathrm{~min}$ at the center of the unperturbed line. The next curves are observed after cw microwave irradiation during $3 \mathrm{~min}$ at the maximum of the antihole of the previous recording. Finally, the antihole stabilizes at a position shifted by $7 \mathrm{mT}$ with respect to the original position. $\mathbf{b}$ Shift of the hole and the antihole versus the number of 3-min cycles of cw microwave irradiation in the $94.9 \mathrm{GHz}$ EPR line of shallow Li-related donors in $\mathrm{ZnO}$ QDs with a diameter of 2.8, 3.4 and $4.2 \mathrm{~nm}$, and that of the shallow $\mathrm{H}$ donors in a $\mathrm{ZnO}$ single crystal. $T=2 \mathrm{~K}$ 

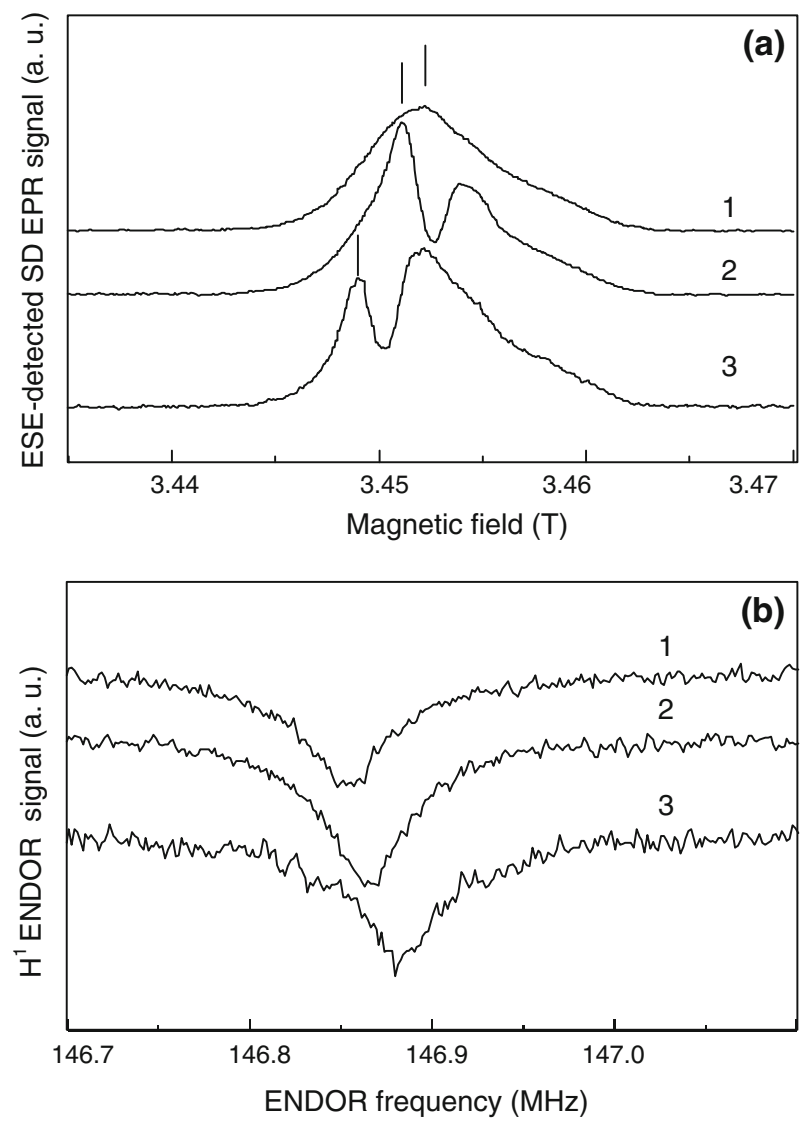

Fig. 12 a Curve (1) represents the unperturbed EPR transition of the shallow donor in ZnO QDs with a diameter of $3.4 \mathrm{~nm}$. Curve (2) is obtained after cw microwave irradiation during $3 \mathrm{~min}$ at the center of the unperturbed EPR line. Curve (3) is recorded after $\mathrm{cw}$ microwave irradiation during $3 \mathrm{~min}$ at the peak of the antihole in curve (2). The bars indicate the positions at which the ENDOR spectra displayed in $\mathbf{b}$ are taken. $v=94.9 \mathrm{GHz}, T=2 \mathrm{~K}$. b ENDOR spectra of ${ }^{1} \mathrm{H}$ nuclei detected in the EPR transition of the shallow donor in $\mathrm{ZnO}$ QDs with a diameter of $3.4 \mathrm{~nm}$ at different magnetic-field positions in the EPR line as indicated in Fig. 12a

with the shallow donor [20]. In particular, the maximum shift of the hole observed in the $\mathrm{ZnO}$ nanoparticles with a diameter of $4.2 \mathrm{~nm}$ is the same as the shift of the EPR line of the shallow donor in the single crystal of $\mathrm{ZnO}$. This is reasonable because it was shown that in the case of the $\mathrm{ZnO}$ single crystal the maximum shift of $2.8 \mathrm{mT}$ could be simulated by considering the complete polarization of all ${ }^{67} \mathrm{Zn}$ spins in a sphere with a radius of about $2.0 \mathrm{~nm}$ [20]. When the diameter of the QD becomes smaller, a considerable increase of the maximum shift the hole is observed (see Fig. 11b). In principle, there are two possible explanations for this observation. First, it is known from previous experiments (Sect. 3.2) that, as a result of the confinement, the density of the wave function at the position of the ${ }^{67} \mathrm{Zn}$ spins in the QD increases when the radius of the particles becomes of the order of the Bohr 
radius or smaller [7]. As a result, the hyperfine interaction increases and thus the shift of the resonance field of the electron spin increases when the nuclear spins become polarized. We have carried out a numerical calculation of the local field in the $\mathrm{ZnO}$ QDs with a diameter of $2.8 \mathrm{~nm}$ produced by a complete polarization of the ${ }^{67} \mathrm{Zn}$ nuclear spins. This calculation was performed in the same way as for the shift of the EPR line observed for the shallow donor in a single crystal of $\mathrm{ZnO}$ [20]. The only differences are that we took into account the effect of confinement of the electronic wave function and the resulting increase of the hyperfine interaction with the ${ }^{67} \mathrm{Zn}$ nuclear spins and that only about 7 shells of $\mathrm{Zn}$ atoms have to be considered in the $2.8 \mathrm{~nm}$ particles. The resulting shift amounts to $3.0-4.0 \mathrm{mT}$, depending somewhat on the estimated value of the Bohr radius. The second possibility is that the ${ }^{1} \mathrm{H}$ nuclear spins in the $\mathrm{Zn}(\mathrm{OH})_{2}$ capping layer also become polarized. When assuming a monolayer of $\mathrm{Zn}(\mathrm{OH})_{2}$ with completely polarized ${ }^{1} \mathrm{H}$ spins and a hyperfine interaction of about $100 \mathrm{kHz}$, as derived from the width of the ENDOR lines in Fig. 2c, we estimate that these polarized ${ }^{1} \mathrm{H}$ nuclear spins produce a maximum hole shift of about $1-2 \mathrm{mT}$ in the $2.8 \mathrm{~nm} \mathrm{ZnO}$ nanoparticles. The conclusion is that the increase of the shift of the hole when reducing the size of the $\mathrm{QD}$ is a result of both the increase of the hyperfine interaction with the polarized ${ }^{67} \mathrm{Zn}$ nuclear spin in the $\mathrm{ZnO}$ core and the polarization of the ${ }^{1} \mathrm{H}$ nuclear spins in the $\mathrm{Zn}(\mathrm{OH})_{2}$ capping layer.

The polarization of the ${ }^{67} \mathrm{Zn}$ nuclear spins in the case of the shallow donor in the single crystal of $\mathrm{ZnO}$ is caused by an Overhauser effect [20, 33]. Here a crossrelaxation process, in which electron spins and nuclear spins undergo flip-flop motions, transfers the electron spin polarization to the nuclear spins upon saturation of the electron spin transition. To make this process efficient, a rapid modulation of the hyperfine interaction is required to induce a fast spin-lattice relaxation rate of the electron spins and a fast cross-relaxation rate, i.e., a fast flip-flop motion. In semiconductors this modulation is provided by the rapid motion of conduction electrons or by the exchange interaction of shallow donors that at high concentrations have a sufficient overlap of their wave function [34, 35]. Since the shallow donors in bulk $\mathrm{ZnO}$ are at low concentration and do not show any sign of a rapid exchange, it was proposed that the modulation of the hyperfine interaction is caused by the fluctuations of the zero-point vibrations of the phonon system. This seems a reasonable suggestion because at the high magnetic field $(10 \mathrm{~T})$ and low temperatures $(5 \mathrm{~K})$, at which the experiments were carried out, the Boltzmann factor is no less than 20 and the spontaneous-emission processes dominate the onephonon-type spin-lattice relaxation of the electron spins.

In the $\mathrm{ZnO}$ nanocrystals, only one donor electron spin is present in a particle and the effect of exchange resulting from an overlap of electronic wave functions can be excluded. Since here the EPR experiments are carried out at $95 \mathrm{GHz}$ in a magnetic field of $3.4 \mathrm{~T}$ and at temperatures of 1.5-2.0 K spontaneous-emission-type processes are also expected to dominate the spin-lattice relaxation. At $95 \mathrm{GHz}$ the typical wavelength of phonons in $\mathrm{ZnO}$ nanocrystals required to induce the onephonon relaxation process of the electron spin of the shallow Li donor is determined by the velocity of sound in this material. When using the value $v=3 \times 10^{3} \mathrm{~ms}^{-1}$ as a reasonable estimate of this velocity one derives that the wavelength of phonons 
at $95 \mathrm{GHz}$, the frequency at which the EPR experiments are carried out, is about $30 \mathrm{~nm}$, i.e., about ten times larger than the average size of the nanoparticles. We thus conclude that the phonons required to induce the one-phonon-type spin-lattice and cross relaxation do not fit into the particles. The remarkable observation is that the spin-lattice relaxation rates observed for the shallow H-donor in the bulk $\mathrm{ZnO}$ single crystal and for the shallow Li-donor in the $\mathrm{ZnO}$ nanocrystal at low temperature are about the same (about $10^{3} \mathrm{~s}^{-1}$ ). To explain the relatively fast onephonon-type spin-lattice relaxation of the shallow Li-donor in the $\mathrm{ZnO}$ nanocrystals we propose that in the dry powder, used in the experiments, the particles are in physical contact with each other and therefore the phonons are not confined to one particle. To check whether this explanation is correct it would be attractive to perform similar experiments on samples of $\mathrm{ZnO}$ nanoparticles dissolved in an organic glass. In such a sample the velocity of sound in the $\mathrm{ZnO}$ particles is much higher than in the glassy host material and one may expect that phonons will reflect at the interfaces, thus leading to a better confinement of the phonons in the $\mathrm{ZnO}$ nanoparticles. A measurement of the spin-lattice relaxation of the shallow donor as a function of the size of the nanoparticles could then be used to confirm the validity of this contention and would allow for a measurement of the distribution of phonon modes in the nanocrystals.

\subsection{Deep-Level Acceptors in ZnO Quantum Dots}

The EPR signal (II) in Fig. 1, which is presented on an enlarged scale in Fig. 13, exhibits a nearly isotropic hyperfine splitting that suggests a hyperfine interaction with a nucleus of spin $I=3 / 2$ with an almost $100 \%$ abundance. This observation favors a Na-related center and indeed the ENDOR spectrum of this EPR signal

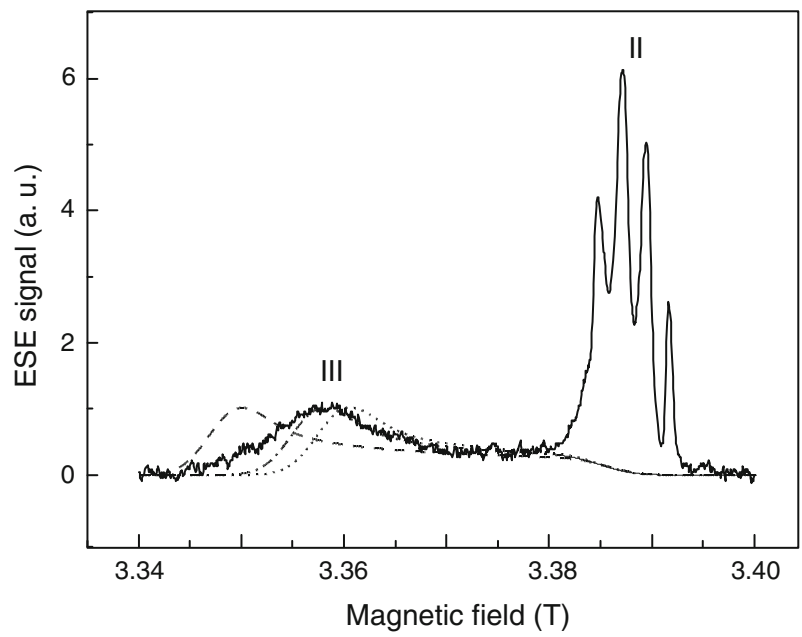

Fig. 13 EPR spectrum of deep acceptors in the core of $\mathrm{ZnO}$ nanocrystals with radii of $1.5 \mathrm{~nm}$ taken at 94.9 GHz and $T=1.5 \mathrm{~K}$. The simulations for the $\mathrm{Li}_{\mathrm{Zn}}$ deep acceptors (dashed line) and for the $\mathrm{V}_{\mathrm{Zn}}$ deep acceptors (dotted line) have been made using the parameters from Refs. [37, 38]. The dashed-dot line is the result of a fitting using $g_{\|}=2.0033$ and $g_{\perp}=2.0205$ 
reveals two transitions at 4.2 and $72.0 \mathrm{MHz}$ as shown in Fig. 14a. These two ENDOR frequencies are given by $v_{\text {ENDOR }}=h^{-1}\left|g_{\mathrm{n}} \beta_{\mathrm{n}} B_{0} \pm A / 2\right|$, when the quadrupole interaction is neglected and where $g_{\mathrm{n}} \beta_{\mathrm{n}} B_{0} / h$ is the Zeeman frequency for sodium. We thus find for the hyperfine constant $A=67.8 \mathrm{MHz}$, which corresponds to a HF splitting of $2.4 \mathrm{mT}$ as observed in the EPR line shape. This splitting of $2.4 \mathrm{mT}$ is about $7 \%$ of the HF constant for free $\mathrm{Na}^{0}$ [36].

The conclusion that this deep Na-related center is located close to or at the $\mathrm{ZnO} / \mathrm{Zn}(\mathrm{OH})_{2}$ interface is drawn from the observation that not only the ENDOR signals of the ${ }^{23} \mathrm{Na}(I=3 / 2)$ nucleus can be observed in the EPR signal (II) but also the ENDOR signal of ${ }^{1} \mathrm{H}(I=1 / 2)$ nuclear spins (see curve (1) in Fig. 14b). The line width of $1.0 \mathrm{MHz}$ is about 8 times larger than that of the ${ }^{1} \mathrm{H}$ ENDOR signals observed in the ESE-detected EPR signal of the shallow donor (see curve (2) in Fig. 14b). This shows that the density of the electronic wave function of the Na-related acceptor is relatively large in the $\mathrm{Zn}(\mathrm{OH})_{2}$ capping layer.

Figure 13 also shows the EPR signal of an additional deep acceptor with a $g$-factor that is typical for deep acceptors in $\mathrm{ZnO}$ bulk material [signal (III) in Figs. 1 and 13]. We note that three types of deep acceptors in $\mathrm{ZnO}$ have similar structures and $g$-values. These centers are, respectively, the substitutional Li or $\mathrm{Na}$ impurity $\left(\mathrm{Li}_{\mathrm{Zn}}\right.$ or $\left.\mathrm{Na}_{\mathrm{Zn}}\right)$, or the $\mathrm{Zn}$ vacancy $\left(\mathrm{V}_{\mathrm{Zn}}\right)[37,38]$. $\mathrm{ZnO}$ has a wurtzite structure, with the $\mathrm{Zn}$ ions surrounded by distorted tetrahedrons of oxygen ions. The hole in the three centers is located on one of the $\mathrm{O}^{2-}$ ligands and one thus has an $\mathrm{O}^{-}$ligand with the unpaired spin located on one of the four $\mathrm{p}$-bonds. There are three non-axial bonds that do not possess perfect axial symmetry and the $g$-tensor of a hole on one of these three oxygen atoms is described by three different principal values $g_{Z}, g_{X}$ and $g_{Y}$. The oxygen in the $c$-axis direction is nonequivalent to the other three oxygen atoms, and the $g$-tensor for a hole on this oxygen atom can be described by $g_{\|}$and $\mathrm{g}_{\perp}$. For the $\mathrm{Li}_{\mathrm{Zn}}$ or $\mathrm{Na}_{\mathrm{Zn}}$ impurities, the preferred site is the axial oxygen atom due to the energy difference between the axial and the non-axial states. For the $\mathrm{V}_{\mathrm{Zn}}$ acceptor only the non-axial state was observed in bulk $\mathrm{ZnO}$ [38].

We have compared the line shape of the EPR signal in Fig. 13 with a simulated curve using the known anisotropy of the $g$-tensor of the deep $\mathrm{Li}_{\mathrm{Zn}}$ and $\mathrm{V}_{\mathrm{Zn}}$ acceptors $[37,38]$ and assuming that the $\mathrm{ZnO}$ nanoparticles are randomly oriented. In Fig. 13 we present the simulations for the EPR line shapes of the deep $\mathrm{Li}_{\mathrm{Zn}}$ acceptors with axial symmetry (dashed line) and for the $\mathrm{Zn}$ vacancy $\left(\mathrm{V}_{\mathrm{Zn}}\right)$ deep acceptor (dotted line). The parameters used for $\mathrm{Li}_{\mathrm{Zn}}$ are $g_{\|}=2.0028, g_{\perp}=2.0253$ and for $\mathrm{V}_{\mathrm{Zn}}$ $g_{Z}=2.0033, g_{X}=2.0192, g_{Y}=2.0188[37,38]$. The dashed-dotted line in Fig. 13 results from a simulation using $g_{\|}=2.0033, g_{\perp}=2.0205$. In all simulations a line width of $5 \mathrm{mT}$ was assumed. From a comparison of the simulated and the experimental curves we conclude that the EPR line in Fig. 13 originates either from the deep $\mathrm{V}_{\mathrm{Zn}}$ or the deep $\mathrm{Li}_{\mathrm{Zn}}$ acceptor, or a combination of these two centers. The simulated EPR spectrum of the $\mathrm{Na}_{Z n}$ acceptor considerably deviates from the experimental spectrum. The shape and position of the signal III in Fig. 13 is slightly sample dependent, and we conclude that the main contribution to the signal is coming from the deep Zn vacancy acceptor which might be introduced during the growth of the nanocrystals. This would thus represent the first direct observation of a vacancy in semiconductor nanocrystals induced during the nanocrystal growth. 

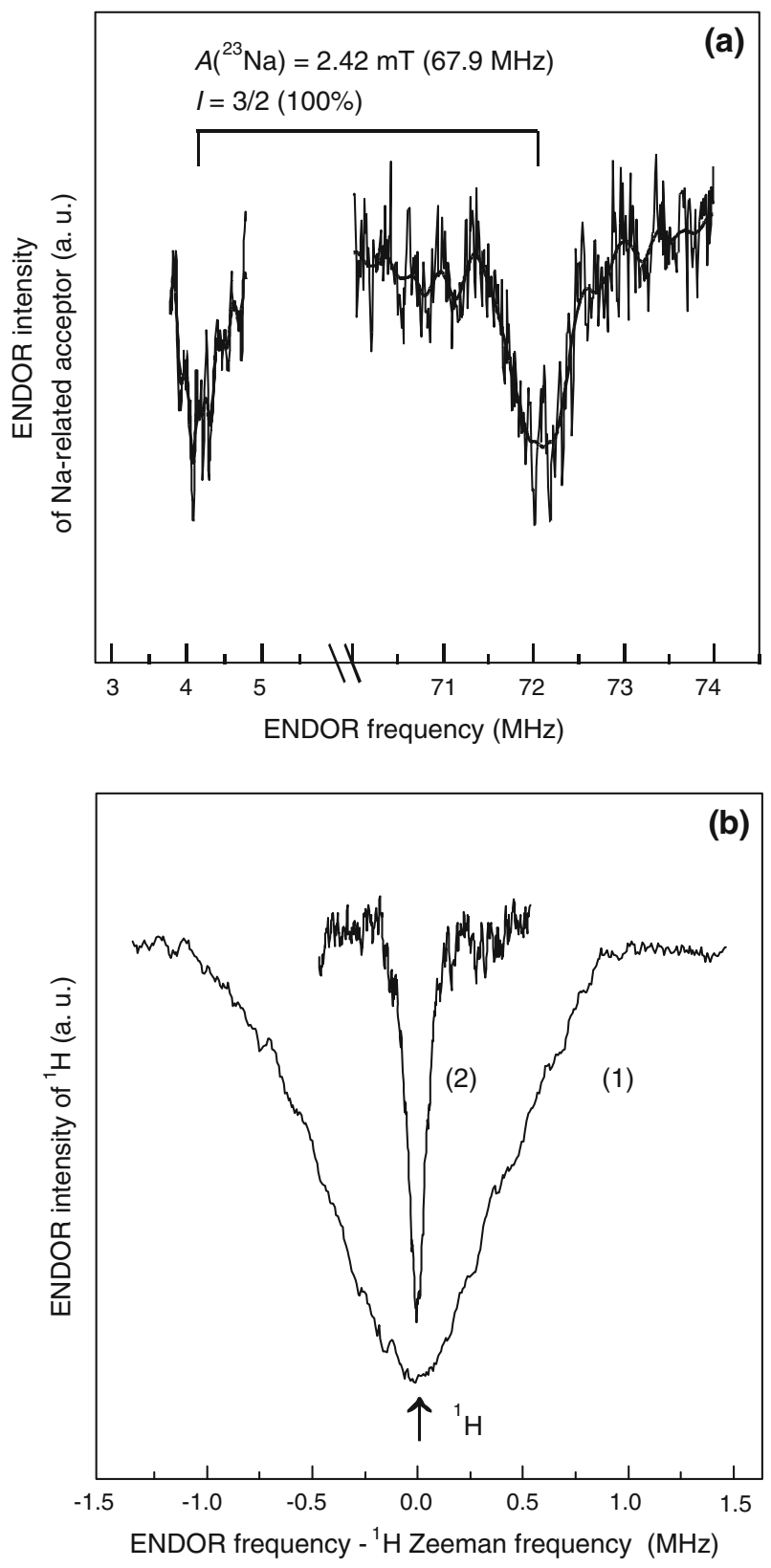

Fig. 14 a ESE-detected ENDOR signals of ${ }^{23} \mathrm{Na}$ nuclei as observed in the EPR signal of a deep Narelated acceptor center in $\mathrm{ZnO}$ quantum dots. The sample has been illuminated during 30 min with UV light prior to the EPR and ENDOR recording $(v=94.9 \mathrm{GHz}, T=2 \mathrm{~K})$. b ENDOR transition of the ${ }^{1} \mathrm{H}$ nuclear spin observed at $94.9 \mathrm{GHz}$ in the EPR signal (II) of the deep Na-related acceptor (1) and in the EPR signal (I) of the shallow Li donor (2) 


\subsection{Donor-Acceptor Pairs in the Confined Structure of $\mathrm{ZnO}$ Quantum Dots}

Exchange-coupled donor-acceptor (or electron-hole) pairs in semiconductors are observable in the EPR spectrum when the electronic wave functions of the donor and acceptor start to overlap significantly. In intentionally doped semiconductors these pairs are difficult to observe because the concentration of donors and acceptors has to be relatively high. An interesting case is formed by the Frenkel pairs (for example in $\mathrm{ZnSe}$ [38]). These pairs consist of a $\mathrm{Zn}$ vacancy and a $\mathrm{Zn}$ interstitial $\left(\mathrm{V}_{\mathrm{Zn}}-\mathrm{Zn}_{\mathrm{i}}\right)$ which stabilize at such a short mutual distance that the exchange interaction, resulting from the overlap of the wave functions, forms the dominant term in the spin Hamiltonian. This spin Hamiltonian then takes the form

$$
H=g_{\mathrm{D}} \beta_{\mathrm{e}} \mathbf{B} \cdot \mathbf{S}_{\mathrm{D}}+g_{\mathrm{A}} \beta_{\mathrm{e}} \mathbf{B} \cdot \mathbf{S}_{\mathrm{A}}+J \mathbf{S}_{\mathrm{D}} \cdot \mathbf{S}_{\mathrm{A}} .
$$

Here the first two terms at the right side describe the Zeeman energies of the donor and acceptor with electron spins $S_{\mathrm{D}}=S_{\mathrm{A}}=1 / 2$ and $g$-factors $g_{\mathrm{D}}$ and $g_{\mathrm{A}} . J$ is the isotropic exchange interaction between the donor and the acceptor. For large values of $J$ such that

$$
|J| \gg\left|g_{\mathrm{D}}-g_{\mathrm{A}}\right| \beta_{\mathrm{e}} B,
$$

where $\beta_{\mathrm{e}}$ and $B$ are the Bohr magneton and the magnetic field, respectively, a single EPR signal arises at the mean $g$-value $g_{\mathrm{P}}=\left(g_{\mathrm{D}}+g_{\mathrm{A}}\right) / 2$ [39-41].

In nominally undoped $\mathrm{ZnO}$ crystals, the EPR signal of interstitial hydrogen shallow donors has recently been observed [18]. The Bohr radius of the hydrogenlike $1 s$-type wave function of this interstitial-hydrogen donor is $a_{\mathrm{D}}=1.5 \mathrm{~nm}$. As yet, only deep acceptors with a localized wave function have been detected in this material. Bohr radius $a_{\mathrm{D}}$ of the donor is much larger than the Bohr radius $a_{\mathrm{A}}$ of the acceptor, the exchange interaction between the shallow donor and the deep acceptor depends exponentially on the donor-acceptor distance, i.e., $J=J_{0} \exp \left(-2 r / a_{\mathrm{D}}\right)$ [42], where $J_{0}$ is the limiting exchange interaction for $r=0$. The value of $J_{0}$ can be estimated from a comparison with the particular case of the self-trapped exciton in $\mathrm{AgCl}$. This self-trapped exciton consists of a hole with a deep level localized on an $\mathrm{Ag}^{+}$site $\left(\mathrm{Ag}^{2+}\right.$ center $)$ and an electron shallowly bound to this center. The Bohr radius of the hydrogen-like $1 s$-type wave function of this electron, $a_{\mathrm{D}}$, is also $1.5 \mathrm{~nm}$ and it was found that $J_{0}=5.37 \mathrm{~cm}^{-1}$ [43]. When using this value of $J_{0}$ we derive that the distance between shallow donors and deep acceptors in $\mathrm{ZnO}$ should be smaller than $2 \mathrm{~nm}$ in order to observe the pair signals. For the typical concentrations of $10^{16}-10^{17} \mathrm{~cm}^{-3}$ of these donors and acceptors in bulk $\mathrm{ZnO}$, their average distance is so large that the probability to find donor-acceptor pairs with an appreciable exchange interaction is negligible.

The probability that donor-acceptor pairs are found in $\mathrm{ZnO}$ QDs is considerably higher than in bulk $\mathrm{ZnO}$ because it is now known that donors and acceptors can be introduced into these materials. In particular, in nanocrystals of $\mathrm{ZnO}$ with radii smaller than $2 \mathrm{~nm}$, that can be routinely produced, one expects that the formation of donor-acceptor pairs will occur. Indeed, we have found evidence for the formation of such pairs as shown in Fig. 15a, where the ESE-detected EPR spectrum of Li-doped $\mathrm{ZnO}$ nanocrystals with an average radius of $1.3 \mathrm{~nm}$ at $94.9 \mathrm{GHz}$ and 
$T=1.6 \mathrm{~K}$ is displayed. In Fig. $15 \mathrm{~b}$ a similarly detected EPR spectrum at $275 \mathrm{GHz}$ and $\mathrm{T}=8 \mathrm{~K}$ is shown. The spectrum in Fig. 15a looks very similar to the one observed at $95 \mathrm{GHz}$ for $\mathrm{ZnO}$ QDs with a radius of $1.7 \mathrm{~nm}$ (Fig. 1). The signal (I) belongs to the shallow Li-related donors and signal (II) to the deep Na-related center. The important difference is that a new EPR signal (I*II) is visible halfway between the signal of the shallow donor (I) and the signal of the deep Na-related

Fig. 15 ESE-detected EPR spectra at $94.9 \mathrm{GHz}$ (a) and at $275 \mathrm{GHz}$ (b) of Li-doped $\mathrm{ZnO}$ quantum dots with an average radius of $1.3 \mathrm{~nm}$ after $\mathrm{UV}$ illumination during $30 \mathrm{~min}$. The signals marked $(I)$ and $(I I)$ arise from an isolated shallow Lirelated donor and a deep acceptor, respectively. A donoracceptor pair formed by the shallow donor and the deep acceptor causes the EPR signal marked by $(I * I I)$. c Variation of the $g$-values of the EPR signals $(I),(I I)$ and $(I * I I)$ as a function of the radius of the $\mathrm{ZnO}$ nanocrystals
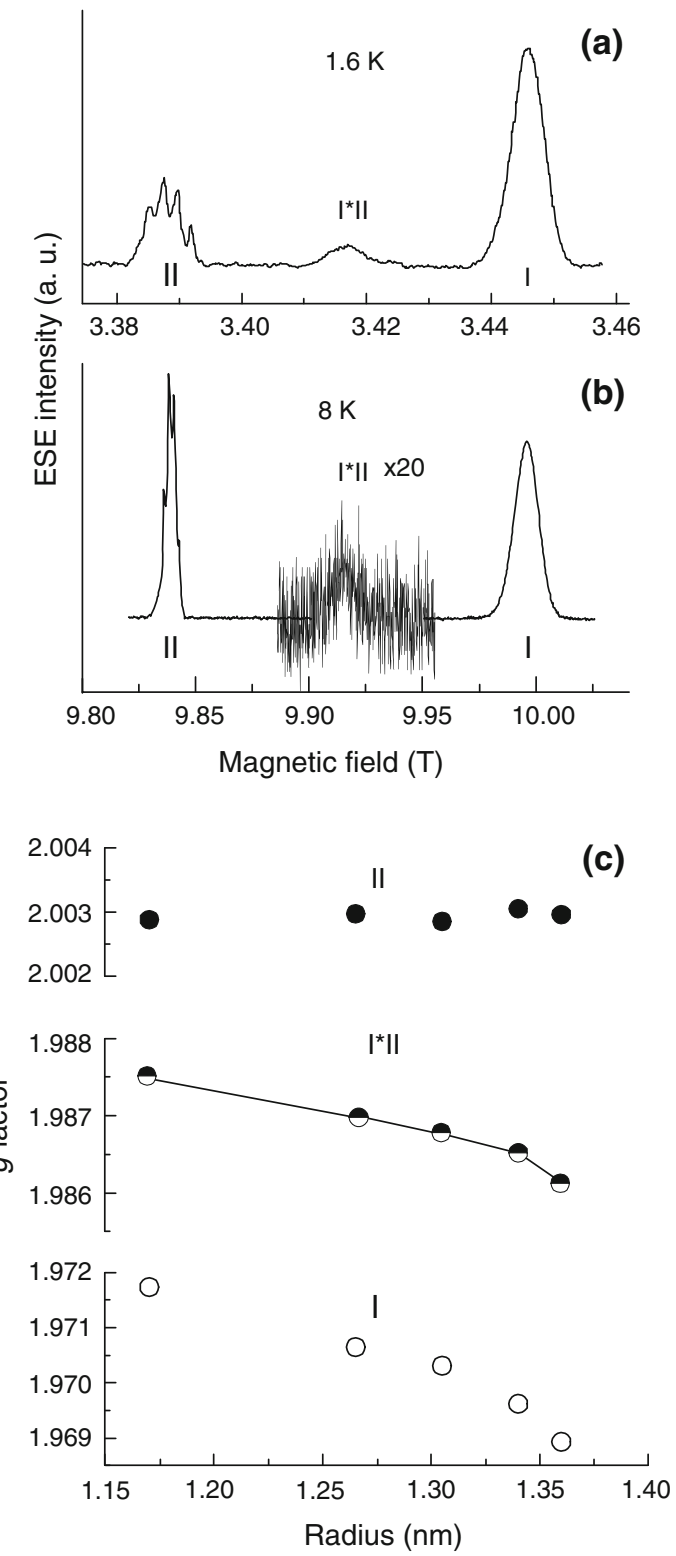


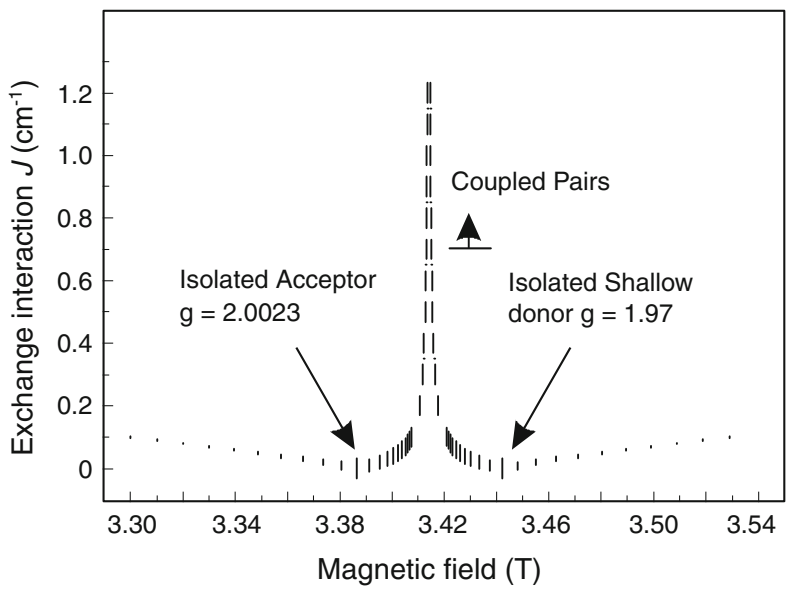

Fig. 16 Predicted positions for the $94.9 \mathrm{GHz}$ EPR transitions of two exchange-coupled particles with spins $S=1 / 2$ as a function of an absolute value of the exchange interaction $J$. The $g$-factors are $g_{\mathrm{A}}=$ 2.0023 for the acceptor and $g_{\mathrm{D}}=1.97$ for the shallow donor. The bar length corresponds to the EPR line intensity

center (II). Signal (I*II) is attributed to an exchange-coupled pair formed by the shallow donor and the deep Na-related center.

The arguments leading to the assignment of signal (I*II) to the exchange-coupled pair of the shallow donor and the deep Na-acceptor are the following. First, its $g$-value $g_{\mathrm{P}}$ is the average of the $g$-values $g_{\mathrm{D}}$ of the shallow Li-donor and the $g$-value $g_{\mathrm{A}}$ of the deep Na-acceptor: $g_{\mathrm{P}}=\left(g_{\mathrm{D}}+g_{\mathrm{A}}\right) / 2$. This is the $g$-value that one predicts for an exchange-coupled donor-acceptor pair when the exchange coupling $J \gg\left(g_{\mathrm{D}}-g_{\mathrm{A}}\right) \beta_{\mathrm{e}} B_{0}$. In Fig. 15b, where the EPR spectrum recorded at $275 \mathrm{GHz}$ is presented [7], the pair signal is again exactly halfway between the signals (I) and (II), which are now separated by an interval that is larger by a factor 275/ $94.9=2.90$. Second, the pair signal is only visible in $\mathrm{ZnO}$ nanoparticles with a radius smaller than $1.5 \mathrm{~nm}$. Apparently, for these particles the exchange interaction, which depends exponentially on the distance, is large enough to create pairs observable in the EPR spectrum.

In Fig. 15c the variation of the $g$-values of signals (I), (II) and (I*II) is presented as a function of the radius of the $\mathrm{ZnO}$ QDs. It is seen that the $g$-value of signal (II) is independent of the size of the nanoparticles, typical for a deep center with a localized wave function. The variation of the $g$-value of signal (I) results from the confinement effect on the wave function of the shallow donor $[3,5]$. It is seen that the variation of the $g$-value of signal (I*II) is half that of the variation of the $g$-value of signal (I), as expected for an exchange-coupled pair formed by the shallow Lirelated donor and the deep Na-related acceptor.

Further support for the assignment of signal (II) as arising from a deeply trapped hole is provided by isochronal annealing experiments [7]. The thermally induced reduction in concentration of the paramagnetic donors and acceptors was monitored by the change in intensity of the EPR signals. The reduction in intensity of signal (I) 
of the shallow donors and that of signal (II) of the Na-related centers were shown to be qualitatively similar. The intensity of the two signals starts to reduce substantially at $50 \mathrm{~K}$. After the annealing at $150 \mathrm{~K}$ the two signals have completely disappeared. The observations lend support to the idea that the thermally released donor electron is captured by the Na-related center making both centers nonparamagnetic. We conclude that the deep Na-related center must have acceptor-like properties. The recombination of the donors and acceptors as observed in the EPRdetected annealing experiments is accompanied by an intense thermoluminescence [7].

The comparison of the EPR/ENDOR and the optical experiments shows that the emission seems to be at least partly due to a charge-transfer transition of an electron from the shallow donor to the deep Na-related trap, which is located close to the $\mathrm{ZnO} / \mathrm{Zn}(\mathrm{OH})_{2}$ interface. This conclusion is drawn from the observation of the ENDOR signal of ${ }^{1} \mathrm{H}$ nuclear spins in the $\mathrm{Zn}(\mathrm{OH})_{2}$ capping layer in the EPR signal of the deep Na-related trap. This shows that the density of the electronic wave function of the Na-related acceptor is relatively large in the $\mathrm{Zn}(\mathrm{OH})_{2}$ capping layer. Further support for this conclusion follows from the effect of saturating the ${ }^{1} \mathrm{H}$ nuclear spin transition on the Na-related EPR signal. The whole line shape was shown [7] to undergo a reduction in intensity, demonstrating that the hyperfine interaction with the ${ }^{1} \mathrm{H}$ nucleus is related to the deep Na-acceptor, and not to an EPR signal of another center that might coincide with the signal of the Na-related acceptor. Before the UV illumination this center is not paramagnetic. Upon aboveband-gap illumination an electron from this center is transferred to the interstitial shallow Li-donor, making both centers paramagnetic. Since we observe a relatively strong hyperfine interaction of this localized Na-related center with a proton of the $\mathrm{OH}^{-}$ligands of $\mathrm{Zn}$ in the capping layer, we expect this complex to be located at or near to the $\mathrm{ZnO} / \mathrm{Zn}(\mathrm{OH})_{2}$ interface. The hole is then most probably located on $\mathrm{Na}$ because about $7 \%$ of the spin density is found on this atom.

As mentioned above, the EPR signals of the isolated shallow donor and the Narelated deep acceptor decrease simultaneously and irreversibly when the temperature is increased. Simultaneously, the EPR signal of the exchange-coupled pair starts to decrease irreversibly at lower temperatures. The signal completely disappears after annealing at $20 \mathrm{~K}$. This could explain the low intensity of the pair signal observed at $275 \mathrm{GHz}$ (Fig. 15b), because here the lowest temperature at which the EPR signal can be observed is $8 \mathrm{~K}$ compared to $1.5 \mathrm{~K}$ at $94.9 \mathrm{GHz}$. The difference in the intensity ratio of the EPR signal of (I) and (II) at 275 and $94.9 \mathrm{GHz}$ (Fig. 15) is due to the difference in the nature of the EPR line broadening. The line width of the shallow donor is caused by $g$-factor anisotropy, which results in an increase of the line width at $275 \mathrm{GHz}$ compared to $94.9 \mathrm{GHz}$ and in a decrease of the signal intensity. The line width of the Na-related hole is dominated by hyperfine interaction, which does not depend on the microwave frequency. As a result, one observes the same intensity at 275 and $94.9 \mathrm{GHz}$.

Figure 16 shows the predicted magnetic-field positions of the EPR transitions at $94.9 \mathrm{GHz}$ of two exchange-coupled spins $S=1 / 2$ of an acceptor with $g_{\mathrm{A}}=2.0023$ and a donor with $g_{\mathrm{D}}=1.97$ as a function of an absolute value of $J$. Such a coupled pair gives rise to a singlet and a triplet states with four possible EPR transitions. The 
bar length in Fig. 16 corresponds to the intensity of the EPR lines. The thick bars indicate the EPR lines of isolated acceptors and isolated shallow donors (i.e., $J=0$ ). The double thick bars in the center of the figure represent the two transitions of a strongly coupled pair. These transitions cannot be resolved in the EPR spectra due to the $5 \mathrm{mT}$ line width of the shallow donors. They appear in the EPR spectrum when $J>0.7 \mathrm{~cm}^{-1}$ and their position does not change appreciably when $J$ is further increased. When $J$ is varied between $5 \times 10^{-3}$ and $0.7 \mathrm{~cm}^{-1}$, the positions of the EPR lines change rapidly. As the value of $J$ is decreased further, the EPR spectrum remains virtually unchanged and consists of two lines corresponding to the isolated donors and acceptors. Thus, EPR signals can be observed only in the case of strong $\left(J>0.7 \mathrm{~cm}^{-1}\right.$ at $\left.94.9 \mathrm{GHz}\right)$ and weak exchange coupling. For intermediate values of $J$, the positions of the EPR lines depend on $J$, and averaging over all possible values of $J$ results in a broad spectrum with a complicated shape and low intensity that is difficult to detect. We further conclude that the sign of $J$ is negative because at $1.2 \mathrm{~K}$ the triplet state is observed.

The most probable position of the shallow interstitial Li-donor, with its large Bohr radius, is near the center of a $\mathrm{ZnO}$ nanocrystal, while the deep Na-related acceptor is localized near the surface of the nanocrystal. Using the expression $J=J_{0} \exp \left(-2 r / a_{\mathrm{D}}\right)$ with $J_{0}=5.37 \mathrm{~cm}^{-1}$ and assuming the radius of the nanoparticle $r=1.3 \mathrm{~nm}$ we can estimate the exchange interaction $J$ to be about $1 \mathrm{~cm}^{-1}$. This is in a good agreement with the value derived from the simulated EPR line positions presented in Fig. 16.

We note that the Na-related surface acceptor is perhaps not the only deep acceptor that can capture the electron of the shallow Li-donor because the intensity of the EPR signal of the deep Na-acceptors was shown [7] to decay faster than that of the shallow Li-donor. As we have shown in Refs. [5, 6], the EPR signal of an additional deep acceptor is observable with $g$-factor that is typical for deep acceptors in $\mathrm{ZnO}$ bulk material. This signal is shown in Fig. 13, where also the signal of the deep Na-related surface acceptor is visible. From a comparison of the simulated and the experimental curves, the EPR line in Fig. 13 was concluded to originate either from the deep $\mathrm{V}_{\mathrm{Zn}}$ or the deep $\mathrm{Li}_{\mathrm{Zn}}$ acceptor, or a combination of these two centers.

The concentration of shallow Li-related donors is estimated to be as low as $10^{16}-10^{17} \mathrm{~cm}^{-3}$, as can be deduced from the intensity of the EPR signals, and consequently only one out of $10^{3}-10^{4}$ particles carries a Li-donor. Based on statistical arguments, we conclude that the concentration of the deep Na-related surface acceptor must be much higher to have an appreciable probability for pair formation in a nanoparticle. Such a high concentration of Na-related acceptors is not unreasonable because it is a surface defect. These deep surface acceptors are probably introduced during the preparation of the nanocrystals by incorporation of $\mathrm{Na}$ impurities from the chemicals, solvents and glassware. Surface adsorption of cations is very likely due to the large surface-to-volume ratio of nanocrystals and would be particularly favored during the precipitation since the $\mathrm{ZnO}$ nanocrystals are negatively charged [21, 22], providing a driving force for the nanocrystals to scavenge cations from solution upon addition of a low dielectric solvent such as heptane. Rinsing with heptane and acetone would probably succeed in removing 
small cations such as $\mathrm{Li}^{+}$from the surface, but not large cations such as $\mathrm{Na}^{+}$. This implies that even small $\mathrm{Na}$ concentrations would be effectively incorporated into the nanoparticles, making it difficult to prepare $\mathrm{Na}^{+}$-free $\mathrm{ZnO} / \mathrm{Zn}(\mathrm{OH})_{2}$ nanocrystals. Indeed, even in $\mathrm{ZnO}$ nanocrystals synthesized in plasticware using compounds and solvents with the lowest commercially available concentration of $\mathrm{Na}$ impurities, the EPR signal of the deep Na-acceptor is still present in comparable strength [7]. Assuming that the incorporation of $\mathrm{Na}^{+}$is nearly quantitative, about $35 \%$ of the $\mathrm{ZnO}$ nanocrystals with an average radius of $1.3 \mathrm{~nm}$ would contain a $\mathrm{Na}^{+}$ion, even at the lowest $\mathrm{Na}$ concentration achievable in our experiments.

The question arises why the EPR signals of the shallow Li-donor and of the Narelated surface acceptor have about equal intensity. We speculate that the majority of the deep Na-related acceptors are not observable in the EPR spectrum because they can only become paramagnetic upon illumination when they can transfer an electron to the Li-related donor. In this respect, we do not believe that the electrons and holes produced upon illumination are captured in different nanocrystals because then the probability for pair formation would be too low for their observation in the EPR spectrum.

Finally, we suggest that similar deep surface acceptors might be present in $\mathrm{CdS}$ and CdSe nanocrystals, which are known to exhibit blinking behavior when a single nanocrystal is optically excited [44]. It is currently thought that such deep acceptors at the surface or in the capping layer of $\mathrm{CdS}$ and $\mathrm{CdSe}$ nanoparticles are essential to explain the lengthening of the "on" periods in the blinking. It would be interesting to study $\mathrm{CdS}$ and $\mathrm{CdSe}$ nanoparticles with EPR and ENDOR techniques to corroborate this suggestion.

\section{Conclusion}

EPR and ENDOR experiments on $\mathrm{ZnO}$ quantum dots having $\mathrm{ZnO} /(\mathrm{ZnOH})_{2}$ core/ shell structures reveal the presence of shallow donors related to interstitial lithium and sodium atoms and substitutional aluminium. The shallow character of the wave function is evidenced by the multitude of ${ }^{67} \mathrm{Zn}$ ENDOR lines and further by the hyperfine interactions with the ${ }^{7} \mathrm{Li},{ }^{23} \mathrm{Na}$ and ${ }^{27} \mathrm{Al}$ nuclei that are much smaller than for atomic lithium, sodium and aluminium.

The results show that one can monitor the change of the electronic wave function of a shallow donor in a $\mathrm{ZnO}$ semiconductor nanoparticle when entering the regime of quantum confinement by using the nuclear spins in the semiconductor nanocrystals as probes. The model based on the effective-mass approximation does not yield an appropriate description of the electronic wave function when the radius of the nanoparticle is reduced below the Bohr radius. We suggest that molecular, cluster-type calculations should be carried out to describe the observed behavior.

The influence of confinement effects on the value of the $g$-factor of SDs in $\mathrm{ZnO}$ and CdS QDs was demonstrated. Dynamic nuclear polarization of nuclear spins in $\mathrm{ZnO}$ quantum dots has been observed, where almost complete polarization of ${ }^{67} \mathrm{Zn}$ and of ${ }^{1} \mathrm{H}$ nuclear spins in the $\mathrm{ZnO} / \mathrm{Zn}(\mathrm{OH})_{2}$ core-shell structure have been obtained 
by saturating the EPR transition of the SD present in the ZnO QDs. DNP manifests itself via the creation of a hole and an antihole in the EPR absorption line of the SD in QDs.

The combination of EPR and optical experiments allowed us to demonstrate that donor-acceptor pairs are formed in the confined structure of $\mathrm{ZnO}$ nanoparticles between the shallow interstitial Li-donor and a deep Na-related acceptor. From ENDOR experiments it is concluded that these deep acceptors are located at the $\mathrm{ZnO} / \mathrm{Zn}(\mathrm{OH})_{2}$ interface. Moreover, we supply arguments that make us believe that deep $\mathrm{Zn}$-vacancy-related deep acceptors are incorporated into the $\mathrm{ZnO}$ nanocrystals during growth.

Acknowledgments We are indebted to D.M. Hofmann, A. Hofstaetter, B.K. Meyer, A. Meijerink, H. Blok, E.J.J. Groenen, and V. Dyakonov for collaboration. This work has been supported by Federal Agency for Science and Innovations, Russia, under the Contracts No. 02.513.12.3031, No. 02.740.11.0108; the Programs of the Russian Academy of Sciences: "Spin-Dependent Effects in Solids and Spintronics"; "Support of Innovations and Elaborations"; "Basic Researches of Nanotechnologies and Nanomaterials" and by the Russian Foundation for Basic Research under Grants No. 09-02-01409 and No. 09-02-00730.

Open Access This article is distributed under the terms of the Creative Commons Attribution Noncommercial License which permits any noncommercial use, distribution, and reproduction in any medium, provided the original author(s) and source are credited.

\section{References}

1. C. de Mello Donegá, P. Liljeroth, D. Vanmaekelbergh, Small 1, 1152 (2005)

2. D.J. Norris, A.L. Efros, S.C. Erwin, Science 319, 1776 (2008)

3. S.B. Orlinskii, J. Schmidt, P.G. Baranov, D.M. Hofmann, C. de Mello Donegá, A. Meijerink, Phys. Rev. Lett. 92, 047603 (2004)

4. D.M. Hofmann, H. Zhou, D.R. Pfisterer, H. Alves, B.K. Meyer, P. Baranov, N. Romanov, C. de Mello Donegá, A. Meijering, S. Orlinskii, H. Blok, J. Schmidt, Phys. Status Solidi C 1, 908 (2004)

5. S.B. Orlinskii, J. Schmidt, E.J.J. Groenen, P.G. Baranov, C. de Mello Donegá, A. Meijerink, Phys. Rev. Lett. 94, 097602 (2005)

6. S.B. Orlinskii, H. Blok, E.J.J. Groenen, J. Schmidt, P.G. Baranov, C. de Mello Donegá, A. Meijerink, Magn. Reson. Chem. 43, S140-S144 (2005)

7. S.B. Orlinskii, H. Blok, J. Schmidt, P.G. Baranov, C. de Mello Donegá, A. Meijerink, Phys. Rev. B 74, 045204 (2006)

8. S.B. Orlinskii, J. Schmidt, P.G. Baranov, D. Rauh, V. Lorrmann, V. Dyakonov, Phys. Rev. B 77, 115334 (2008)

9. S.B. Orlinskii, J. Schmidt, P.G. Baranov, C. de Mello Donegá, A. Meijerink, Phys. Rev. B 79, 165316 (2009)

10. B.K. Meyer, H. Alves, D.M. Hofmann, W. Kriegseis, D. Forster, F. Bertram, J. Christen, A. Hoffmann, M. Straßburg, M. Dworzak, U. Haboeck, A.V. Rodina, Phys. Status Solidi B 241(2), 231 (2004)

11. K.M. Whitaker, S.T. Ochsenbein, V.Z. Polinger, D.R. Gamelin, J. Phys. Chem. C 112, 14331 (2008)

12. S.T. Ochsenbein, Y. Feng, K.M. Whitaker, E. Badaeva, W.K. Liu, X. Li, D.R. Gamelin, Nat. Nanotechnol. 4, 681 (2009)

13. W.J.E. Beek, M.M. Wienk, R.A.J. Janssen, Adv. Funct. Mater. 16, 1112 (2006)

14. C. Gonzales, D. Block, R.T. Cox, A. Hervé, J. Cryst. Growth 59, 357 (1982)

15. D. Block, A. Hervé, R.T. Cox, Phys. Rev. B 25, 6049 (1982)

16. N.Y. Garces, N.C. Giles, L.E. Halliburton, G. Cantwell, D.B. Eason, D.C. Reynolds, D.C. Look, Appl. Phys. Lett. 80, 1334 (2002) 
17. M.T. Bennebroek, O.G. Poluektov, A.J. Zakrzewski, P.G. Baranov, J. Schmidt, Phys. Rev. Lett. 74, 442 (1995)

18. D.M. Hofmann, A. Hofstaetter, F. Leiter, H. Zhou, F. Henecker, B.K. Meyer, S.B. Orlinskii, J. Schmidt, P.G. Baranov, Phys. Rev. Lett. 88, 045504 (2002)

19. S.B. Orlinskii, J. Schmidt, P.G. Baranov, M. Bickermann, B.M. Epelbaum, A. Winnacker, Phys. Rev. Lett. 100, 256404 (2008)

20. H. Blok, S.B. Orlinski, J. Schmidt, P.G. Baranov, Phys. Rev. Lett. 92, 047602 (2004)

21. E.A. Meulenkamp, J. Phys. Chem. B 102, 5566 (1998)

22. A. van Dijken, E.A. Meulenkamp, D. Vanmaekelbergh, A. Meijerink, J. Phys. Chem. B 104, 1715 (2000)

23. Z. Hu, G. Oskam, P.C. Searson, J. Colloid Interface Sci. 263, 454 (2003)

24. V. Noack, A. Eychmuller, Chem. Mater. 14, 1411 (2002)

25. C. de Mello Donegá, A.A. Bol, A. Meijerink, J. Lumin. 96, 87 (2002)

26. J.A.J.M. Disselhorst, H.J. van der Meer, O.G. Poluektov, J. Schmidt, J. Magn. Reson. A 115, 183 (1995)

27. H. Blok, J.A.J.M. Disselhorst, H. van der Meer, S.B. Orlinskii, J. Schmidt, J. Magn. Reson. 173, 49 (2005)

28. W.B. Mims, in Electron Paramagnetic Resonance, ed. by S. Geschwind (Plenum, New York, 1972), pp. 263-351

29. C.H. Park, S.B. Zhang, S.-H. Wei, Phys. Rev. B 66, 073202 (2002)

30. S. Öğüt, R. Burdick, Y. Saad, J.R. Chelikowsky, Phys. Rev. Lett. 90, 127401 (2003)

31. D.V. Melnikov, J.R. Chelikowsky, Phys. Rev. Lett. 92, 046802 (2004)

32. A.V. Rodina, A.L. Efros, M. Rosen, B.K. Meyer, Mater. Sci. Eng. C 19(1-2), 435 (2002)

33. A.W. Overhauser, Phys. Rev. 89, 689 (1953)

34. G. Denninger, D. Reiser, Phys. Rev. B 55, 5073 (1997)

35. V. Dyakonov, G. Denninger, Phys. Rev. B 46, 5008 (1992)

36. J.R. Morton, K.F. Preston, J. Magn. Reson. 30, 577 (1978)

37. O.F. Schirmer, D. Zwingel, Solid State Commun. 8, 1559 (1970)

38. A.L. Taylor, G. Filipovich, G.K. Lindeberg, Solid State Commun 8, 1359 (1970)

39. F.C. Rong, W.A. Barry, J.F. Donegan, G.D. Watkins, Phys. Rev. B 54, 7779 (1996)

40. W.A. Barry and G.D. Watkins, Phys. Rev. B 54, 7789 (1996)

41. N.M. Atherton, Principles of Electron Spin Resonance (Ellis Horwood, PTR Prentice-Hall, New York, 1993)

42. R.T. Cox, J.J. Davies, Phys. Rev. B 34, 8591 (1986)

43. O.G. Poluektov, M.C.J.M. Donckers, P.G. Baranov, J. Schmidt, Phys. Rev. B 47, 10226 (1993)

44. M. Nirmal, B.O. Dabbousi, M.G. Bawendi, J.J. Macklin, J.K. Trautman, T.D. Harris, L.E. Brus, Nature 383, 802 (1996) 